\title{
Changes in the phenolic compositions of Elaeagnus umbellata and Sambucus lanceolata after in vitro gastrointestinal digestion and evaluation of their potential anti-diabetic properties
}

\author{
Vítor Spínola $^{\mathrm{a}}$, Joana Pinto ${ }^{\mathrm{a}}$, Eulogio J. Llorent-Martínez ${ }^{\mathrm{b}}$, Paula C. Castilho ${ }^{\mathrm{a}, *}$ \\ ${ }^{a}$ CQM - Centro de Química da Madeira, Universidade da Madeira, Campus da Penteada, 9020-105 Funchal, Portugal \\ ${ }^{\mathrm{b}}$ Department of Physical and Analytical Chemistry, Faculty of Experimental Sciences, University of Jaén, Campus Las Lagunillas, E-23071 Jaén, Spain
}

\section{A R T I C L E I N F O}

\section{Keywords:}

Elaeagnus umbellata

Sambucus lanceolata

Phenolic composition

Gastrointestinal digestion simulation

Digestive enzymes inhibition

Aldose reductase

Protein glycation

Antioxidant activity

\begin{abstract}
A B S T R A C T
In this work, the phenolic composition of $E$. umbellata leaves and berries is reported. Berries were rich in flavonols, whereas leaves presented abundant flavonols and ellagitannins. Then, the enzyme-inhibitory properties, anti-glycation and antioxidant activities of E. umbellata and Sambucus lanceolata (its phenolic profile has been already established in a previous work) were tested by several in vitro assays and compared. The simulated gastrointestinal digestion resulted in a decrease on their phenolic composition. Nevertheless, both species still had the ability to inhibit aldose reductase activity and protein glycation and scavenge free radicals at the end of the process. Thus, the potential beneficial effects of $E$. umbellata and $S$. lanceolata seems to be kept to some extent after passage throughout the digestive system. Altogether, this study provides further insight into investigation of these species as dietary sources of bioactive compounds to lower the risk of type-2 diabetes and obesity.
\end{abstract}

\section{Introduction}

The incidence of diabetes mellitus (DM) is epidemically increasing all over the world and is estimated to reach almost 600 million people by the year of 2035 (Grewal, Bhardwaj, Pandita, Lather, \& Sekhon, 2016; Nazir et al., 2018). The lack of physical activity and excessive intake of carbohydrate and/or fat-rich foods has contributed to the prevalence of type-2 diabetes (T2DM) (90-95\% of all DM cases) (Ho et al., 2017; Podsędek, Majewska, Redzynia, Sosnowska, \& Koziołkiewicz, 2014; Worsztynowicz, Napierała, Białas, Grajek, \& Olkowicz, 2014). The main signs of this metabolic condition is hyperglycaemia (raised blood sugar levels), which is due to deficiency or abnormalities in insulin secretion and/or the insensitivity of target tissues to the metabolic action of this hormone (Ho, Kase, Wangensteen, \& Barsett, 2017; You, Chen, Wang, Luo, \& Jiang, 2011). If remained untreated, chronic hyperglycaemia can lead to several complications, affecting the cardiovascular, renal, neurological and visual systems (Nazir et al., 2018; Yeh, Hsia, Lee, \& Wu, 2017).

Among fruits, berries (or berry fruits) have been recently recognized as "superfoods" due to their unique and appreciable phenolic contents and associated health benefits (antioxidant, anti-inflammatory, antidiabetic, etc) (Edirisinghe \& Burton-Freeman, 2016; Harris et al., 2014; Ho, Nguyen, et al., 2017; McDougall \& Stewart, 2005). Their anti-diabetic effects include regulation of glucose digestion and absorption, reduction of oxidative stress and inflammation, inhibition of the polyol pathway, prevention of protein glycation, etc. (Edirisinghe \& BurtonFreeman, 2016; Harris et al., 2014; Nazir et al., 2018). Hence, berries consumption can constitute an easy, safe and cost-effective dietary approach to suppress hyperglycaemia and its associated complications (Edirisinghe \& Burton-Freeman, 2016; McDougall, Kulkarni, \& Stewart, 2008; McDougall \& Stewart, 2005). Infusions and decoctions of leaves from berry-producing plants are also prepared due to their medicinal

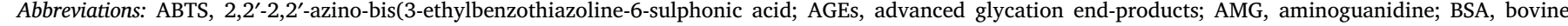

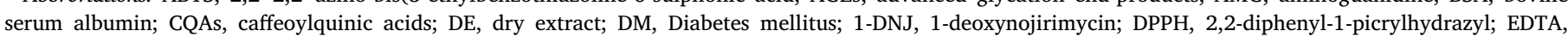

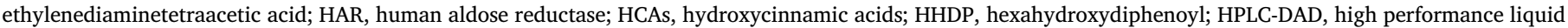

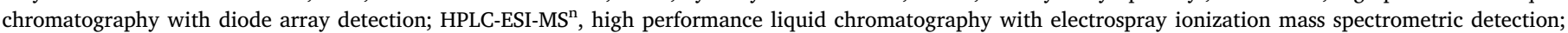

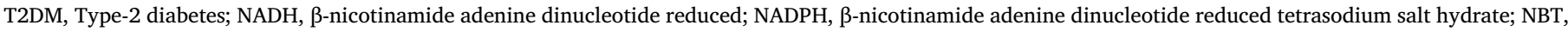

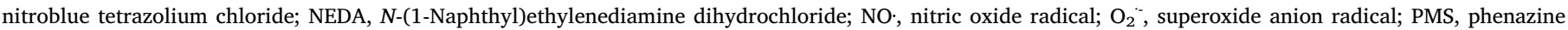

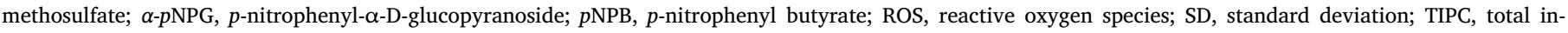
dividual phenolic content

* Corresponding author.

E-mail address: castilho@uma.pt (P.C. Castilho).
} 
value (Ferlemi \& Lamari, 2016). Generally, they possess higher and richer phenolic composition than berries (Kim, Lim, \& Yang, 2016; Ozen, Yenigun, Altun, \& Demirtas, 2017; Spínola, Pinto, \& Castilho, 2018; Spínola, Pinto, Llorent-Martínez, Tomás, \& Castilho, 2019), being considered as alternative sources for the development of food supplements and nutraceuticals (Ferlemi \& Lamari, 2016).

To achieve any beneficial health effects, phenolic compounds must be released from the berries along the gastrointestinal tract, remain in a bioactive form, be absorbed from the gut into the bloodstream, and then be delivered to the appropriate location within the body (Bermúdez-Soto, Tomás-Barberán, \& García-Conesa, 2007; Liang et al., 2012; Tagliazucchi, Verzelloni, Bertolini, \& Conte, 2010). Due to the low-cost, simplicity, rapidity and reproducibility, in vitro digestion models have been largely used as a first approach to simulate the physiological conditions occurring in human digestion (Correa-Betanzo et al., 2014; Guerra et al., 2012; Liang et al., 2012). The obtained results provide a useful knowledge about the possible effects of $\mathrm{pH}$ and digestive enzymes, food matrix and interactions with other components in the stability and bioavailability of phenolic compounds during gastrointestinal digestion (Bermúdez-Soto et al., 2007).

Elaeagnus umbellata Thunb (Elaeagnaceae), commonly known as Autumn olive or Japanese silverberry, is an indigenous species to Central Asia (China, India, Afghanistan, Korea, Pakistan) (Nazir et al., 2018; Ozen et al., 2017) that was introduced to Madeira Island (Portugal). It is described as a deciduous shrub or small tree (typically up to 3.5 to 5.5 . $\mathrm{m}$ tall) that grows a small, tasty fleshy drupe, which ripens to red, dotted with silver or brown colour (Ishaq, Rathore, Sabir, \& Maroof, 2015; Khattak, 2012). Berries are very common in most Asian countries diet, being also used to produce beverages, sauces, jams, etc. (Ozen et al., 2017; Pei, Yu, Bruno, \& Bolling, 2015). Sambucus lanceolata R. Br. in Buch (Adoxaceae) (Madeira elderberry) or "sabugueiro" is a small tree or shrub, up to $7 \mathrm{~m}$ tall, endemic to Madeira Island (Portugal). Berries are small yellowish round edible fruits that get darkpurple when ripe in the late Summer (Pinto et al., 2017).

In view of the reported anti-diabetic benefits of berries and leaves (Edirisinghe \& Burton-Freeman, 2016; Ferlemi \& Lamari, 2016), the aim of this research was to evaluate the in vitro inhibitory activities of both species on $\alpha$-glucosidase, $\alpha$-amylase, pancreatic lipase and aldose reductase enzymes, as well as their anti-glycation and antioxidant activities. The impact of simulated gastrointestinal digestion on the stability of phenolic compounds and changes on their potential bioactivities was also investigated.

\section{Materials and methods}

\subsection{Chemicals and reagents}

All reagents met the quality norms required for analytical grade reagents. Acarbose, aminoguanidine hydrochloride (AMG), ammonium sulfate, bovine serum albumin (BSA), $\alpha$-amylase from porcine pancreas (type VI-B), intestinal acetone powder from rat source of $\alpha$-glucosidase, lipase (type II; from porcine pancreas), DL-glyceraldehyde, $\beta$-mercaptoethanol, mucin (type II; from porcine stomach), $\beta$-nicotinamide adenine dinucleotide reduced (NADH), $N$-(1-Naphthyl)ethylenediamine dihydrochloride, phenazine methosulfate, pancreatin (porcine pancreas), pepsin (porcine gastric mucosa), porcine bile extract, $p$-nitrophenyl- $\alpha$-D-glucopyranoside, $p$-nitrophenyl butyrate, and orlistat were all acquired from Sigma-Aldrich (St. Louis, MO, USA). Human aldose reductase was purchased from Prozomix (Northumberland, UK) and $\beta$-nicotinamide adenine dinucleotide reduced tetrasodium salt hydrate from Calbiochem (MA, USA). 1-Deoxynojirimycin (1-DNJ) was acquired from Biopurify phytochemicals LTD (Chengdu, China). Nitroblue tetrazolium chloride was purchased from Acros Organics (Geel, Belgium). Other chemicals and reagents used in this study are detailed in the Supplementary Material.

\subsection{Sample preparation and extraction of phenolic compounds}

Plant material (leaves and berries) of E. umbellata and S. lanceolata were collected in different areas of Madeira Island (Portugal) in October 2014 and specimens were stored at Madeira Botanical Garden Herbarium (Funchal, Madeira Island) (Table S1 - Supplementary Material).

For analysis, samples were separated into leaves and fruits (fully ripe), destemmed, washed, lyophilized (Alpha 1-2 LD plus freeze dryer, CHRIST), ground to powder using a mechanic grinder, and stored at $-20^{\circ} \mathrm{C}$. Extraction of phenolic compounds followed a previous procedure (Pinto et al., 2017). In brief, $1 \mathrm{~g}$ of dry material was extracted with methanol in an ultra-sonic bath (Bandelin Sonorex, Germany) at $35 \mathrm{~Hz}$ and $200 \mathrm{~W}$ for $60 \mathrm{~min}$. For berry fruits, an extraction solution composed of $\mathrm{MeOH} / \mathrm{H}_{2} \mathrm{O}(80: 20, v / v)$ acidulated with $7 \%$ acetic acid was used. After sonication, solutions were filtered through Whatman No.1 filter papers, concentrated to dryness in a rotary evaporator (at $40^{\circ} \mathrm{C}$ ), and the resulting dry extracts (DE) were stored at $4^{\circ} \mathrm{C}$ until further analysis.

\subsection{Simulation of gastrointestinal digestion}

The same static model previously reported (Pinto et al., 2017) was used to simulate, sequentially, mouth, stomach and small intestine digestion. For this, artificial gastrointestinal juices (saliva, gastric, duodenal, bile) were prepared freshly (Table S2-Supplementary Material). In $50 \mathrm{~mL}$ Falcon tubes, $2 \mathrm{~g}$ of lyophilized berries or leaves were mixed, separately, with $4 \mathrm{~mL}$ of salivary juice and immersed in a water bath $\left(37^{\circ} \mathrm{C}\right)$ with agitation $(150 \mathrm{rpm})$, protected from light (for $\left.5 \mathrm{~min}\right)$. Then, $10 \mathrm{~mL}$ of gastric juice was added to the mixture and further incubated for an additional $2 \mathrm{~h}$. After this period, $10 \mathrm{~mL}$ of duodenal and $4 \mathrm{~mL}$ of bile juices were added and the solution was mixed for $2 \mathrm{~h}$. Two independent replicated digestions were performed for each sample. At the end of the digestion simulation, samples were centrifuged $(4000 \mathrm{rpm}$, $10 \mathrm{~min}$ ). Then, the supernatant was recovered and filtered through Whatman No.1 filter papers, lyophilized and submitted to extraction (as described in the previous section). The liquid extracts were concentrated to dryness and stored at $4{ }^{\circ} \mathrm{C}$ pending its use.

\subsection{Analysis of phenolic compounds}

The chromatographic analysis was carried out on a Dionex ultimate 3000 series instrument (Thermo Scientific Inc.) equipped with a binary pump, an autosampler, a column compartment (kept at $30^{\circ} \mathrm{C}$ ) and a diode-array detector (DAD) coupled to a Bruker Esquire model 6000 ion-trap mass spectrometer (Bremen, Germany). Separation was performed on a Phenomenex Gemini $\mathrm{C}_{18}$ column $(5 \mu \mathrm{m}, 250 \times 3.0 \mathrm{~mm}$ i.d.) using the same conditions reported previously (Pinto et al., 2017). Water/formic acid $(0.1 \%, v / v)(\mathrm{A})$ and $\mathrm{CH} 3 \mathrm{CN}$ (B) was used as mobile phase at a flow rate of $0.4 \mathrm{~mL} \mathrm{~min}^{-1}$. The gradient program was set as: $20 \%$ A (0 min), $25 \%$ A (10 min), 25\% A (20 min), 50\% A (40 min), $100 \%$ A ( $42-47 \mathrm{~min})$ and $20 \%$ A (49-55 min). Mass spectrometry analysis was performed in negative and positive mode and scan range was set at $m / z 100-1000$ with speed of $13,000 \mathrm{Da} / \mathrm{s}$. The conditions of ESI were as follows: drying and nebulizer gas $\left(\mathrm{N}_{2}\right)$ flow rate and pressure, $10 \mathrm{mLmin}^{-1}$ and $50 \mathrm{psi}$; capillary temperature, $325^{\circ} \mathrm{C}$; capillary voltage, $4.5 \mathrm{keV}$; collision gas $(\mathrm{He}) \mathrm{MS}^{\mathrm{n}}$ data was made in auto $\mathrm{MS}^{\mathrm{n}}$ mode, with isolation width of pressure and energy, $1 \times 10^{-5} \mathrm{mbar}$ and $40 \mathrm{eV}$. The acquisition of $4.0 \mathrm{~m} / \mathrm{z}$, and a fragmentation amplitude of $1.0 \mathrm{~V}\left(\mathrm{MS}^{\mathrm{n}}\right.$ up to $\left.\mathrm{MS}^{4}\right)$.

\subsection{Quantification of main polyphenols}

For the determination of polyphenols in the analysed extracts, caffeic acid, quercetin, apigenin, (+)-catechin, and ellagic acid standards were used for the relative quantification of hydroxycinnamic acids (HCAs), flavonols, flavones, flavan-3-ols and ellagitannins, respectively 
(Pinto et al., 2017). The calibration curves $\left(5-100 \mathrm{mg} \mathrm{L}^{-1}\right)$, obtained plotting peak area versus concentration, had $\mathrm{R}^{2} \geq 0.990$ in all cases. Total individual phenolic content (TIPC) was defined as the sum of the relative concentrations of phenolic compounds in extracts.

\subsection{Enzyme inhibition and protein glycation assays}

$\alpha$-Glucosidase, $\alpha$-amylase, lipase, aldose reductase and BSA-glycation inhibition assays were determined using the procedures detailed in the Supplementary Material. The obtained inhibitory activities were expressed as the $\mathrm{IC}_{50}$ value $\left(\mathrm{mg} \mathrm{mL}^{-1}\right.$ of dry extract, $\mathrm{DE}$ ).

\subsection{In vitro antioxidant assays}

The antioxidant activity of analysed extracts was determined by ABTS $^{*+}$, DPPH', nitric oxide (NO) and superoxide $\left(\mathrm{O}_{2}{ }^{-}\right)$in vitro assays, following the same procedures detailed in the Supplementary Material. The results were expressed as $\mu \mathrm{mol}$ of Trolox equivalent (TE) $\mathrm{g}^{-1} \mathrm{DE}$, based on the Trolox calibration curve.

\subsection{Statistical analysis}

Statistical analysis was performed using SPSS Statistics software v.20 (IBM SPSS Statistics for Windows, IBM Corp., USA). Data of all analysis, in triplicate, are expressed as mean \pm standard deviation. A one-way analysis of variance (ANOVA) was performed to determine whether there are any statistically significant differences among parameters experimentally determined, followed by Tukey's HSD post-hoc test. A value of $p<.05$ was considered statistically significant.

\section{Results and discussion}

\subsection{Phytochemical screening}

In this work, the analysis of phytochemical composition in the extracts of E. umbellata (berries and leaves) was carried out by HPLC-ESI$\mathrm{MS}^{\mathrm{n}}$ using negative and positive ionization modes (the latter used for confirmation purposes). Compounds were numbered by their order of elution and this numeration was kept identical for both extracts. The characterization of compounds (Table 1) was assigned based on the comparison of the experimental mass spectra with data from literature (listed in the Supplementary Material). The discussion of the characterization of compounds is explained in detail in the Supplementary Material. A total of 94 phytochemicals were tentatively identified, including flavonoids, phenolic acids, ellagitannins, organic acids, terpenoids and saccharides. The base peak chromatograms (BPCs) of the methanol extracts are shown in Fig. S1 (Supplementary Material).

The composition of $S$. lanceolata methanolic extracts (berries and leaves) has been previously characterised by our work group (Pinto et al., 2017). Seventy-seven phytochemicals were described, namely phenolic compounds (flavonoids and phenolic acids) and other substances (organic acids, oligosaccharides, lignans, terpenoids and fatty acids).

\subsection{Phenolic composition determination}

Thirty-six phenolic compounds were quantified by HPLC-DAD in $E$. umbellata extracts (Table 2).

TIPC varied between 5.56 and $40.35 \mathrm{mg} \mathrm{g}^{-1} \mathrm{DE}$, corresponding to berries and leaves, respectively (Table 2). Flavonols (78.8\%) were the most abundant compounds in berries, followed by ellagitannins (9\%), flavones $(7.8 \%)$, flavan-3-ols $(2.7 \%)$ and HCAs $(1.8 \%)$. A different composition was verified in the case of leaves: ellagitannins $(56.8 \%)>$ flavonols $(40.7 \%)>$ HCAs $(5.2 \%)>$ flavan-3-ols $(2.1 \%)$.

Kaempferol-O-(coumaroyl)hexoside (87) was the dominant compound in berries (37.2\%) (Table 2). Quercetin-O-pentoside (49) (8.4\%),
kaempferol-O-(coumaroyl)hexoside (89) (6.6\%) and kaempferol-Ohexoside (73) (6.1\%) were also relevant. Bis-hexahydroxydiphenoyl (HHDP)-O-hexoside (17) (26.7\%) was the main compound in leaves, followed by galloyl-HHDP-O-hexoside (21) (15.5\%), quercetin-O(pentosyl)hexoside (51) (12.5\%) and isorhamnetin-O-(pentosyl)hexoside $(9.30 \%)$. Qualitative and quantitative differences were observed in the literature. It was determined that $E$. umbellata berries were composed mainly by phenolic acids (HCAs and hydroxybenzoic acids) (Ishaq et al., 2015; Ozen et al., 2017), while other authors reported flavan-3-ols (catechin and its polymers) (Pei et al., 2015). Nazir and coworkers (Nazir et al., 2018) stated chlorogenic acid, quercetin, and epigallocatechin-gallate as major phenolic compounds of berries. Gallic acid and kaempferol (Kim et al., 2016) and hesperidin, rutin, neohesperidin, and ellagic acid were dominant in the leaves extracts (Ozen et al., 2017). Observed discrepancies could be related to different edaphoclimatic conditions and/or post-harvest procedures (sample preparation, solvents, extraction methodologies, etc.).

In comparison with $E$. umbellata, extracts of $S$. lanceolata showed higher and lower TIPC contents for berries and leaves, respectively (Fig. 1). S. lanceolata berries were composed mainly by anthocyanins (88.6\%), which were absent in E. umbellata (confirmed by the positive ionization mode and UV-spectrum at $520 \mathrm{~nm}$ ). HCAs (57.1\%) and flavonols $(42.9 \%)$ were dominant in the leaves of $S$. lanceolata (Pinto et al., 2017).

After simulated digestion, qualitative and quantitative differences were found in $E$. umbellata in relation to native values $(p<.05)$ (Table 2). Berries components were more unstable than leaves ones (reductions of $71.05 \%$ and $62.24 \%$ of TIPC, respectively). Flavonols and flavones from berries suffered degradations of $67.28 \%$ and $59.30 \%$, respectively. Kaempferol- $O$-(coumaroyl)hexoside (87) and quercetin- $O$ pentoside (49) contents were reduced by $52.70 \%$ and $60.00 \%$, respectively. In the case of leaves, HCAs content showed the highest loss (90.20\%), followed by ellagitannins (62.59\%) and flavonols (43.63\%). Main compounds, bis-HHDP-O-glucose (17), quercetin-O-pentosyl (hexoside) (51) and galloyl-HHDP-O-hexoside (21) showed decreases of $62.51 \%, 43.10 \%$ and $61.68 \%$, respectively, upon simulated digestion. Information regarding the stability of phenolic compounds in the gastrointestinal tract occurring in E. umbellata is lacking in the literature. However, a similar trend was verified for other berry-producing species, with losses of TIPC ranging from $23 . \%$ to $80.5 \%$ (Bermúdez-Soto et al., 2007; Correa-Betanzo et al., 2014; Olejnik et al., 2016; Zhang et al., 2016). A reduction of TIPC was also observed for berries and leaves of S. lanceolata ( $81.8 \%$ and $61.5 \%$, respectively) upon simulated digestion (Pinto et al., 2017). Anthocyanins content was highly affected $(-87.2 \%)$ in berries; HCAs (35.8-54.9\%) and flavonoids (56.3-70.6\%) contents were also decreased in the leaves.

The loss of phenolic content is mainly attributed to the chemical conditions during intestinal digestion, since most phenolic compounds are highly sensitive to the mild alkaline conditions and their structures may undergo modifications (hydrolysis, conversion/breakdown, etc.) (Bermúdez-Soto et al., 2007; Huang, Sun, Lou, Li, \& Ye, 2014; Zhang et al., 2016). Anthocyanins are generally considered to be the most unstable compounds at neutral or slightly basic pH (Bermúdez-Soto et al., 2007; Correa-Betanzo et al., 2014; Liang et al., 2012; Olejnik et al., 2016) due to the formation of the colorless chalcone pseudo-base resulting in the destruction of the anthocyanins chromophore (Liang et al., 2012; Pinto et al., 2017; Tagliazucchi et al., 2010). The nonanthocyanin phenolic compounds are more stable at the intestinal alkaline pH value (Liang et al., 2012; Tagliazucchi et al., 2010), which has been reported to cause oxidation and racemization of molecules (Bermúdez-Soto et al., 2007). Interactions between phenolic compounds and other components, such as digestive enzymes, pancreatin bile salts, or even with other food matrix components (proteins, lipids, fibers) can also limit their bioavailability (Bermúdez-Soto et al., 2007; Zhang et al., 2016). 
Table 1

Characterization of phytochemicals of $E$. umbellata methanolic extracts by HPLC-ESI ${ }^{-} / \mathrm{MS}^{\mathrm{n}}$. A more detailed explanation about the identification of compounds is presented in the Supplementary material.

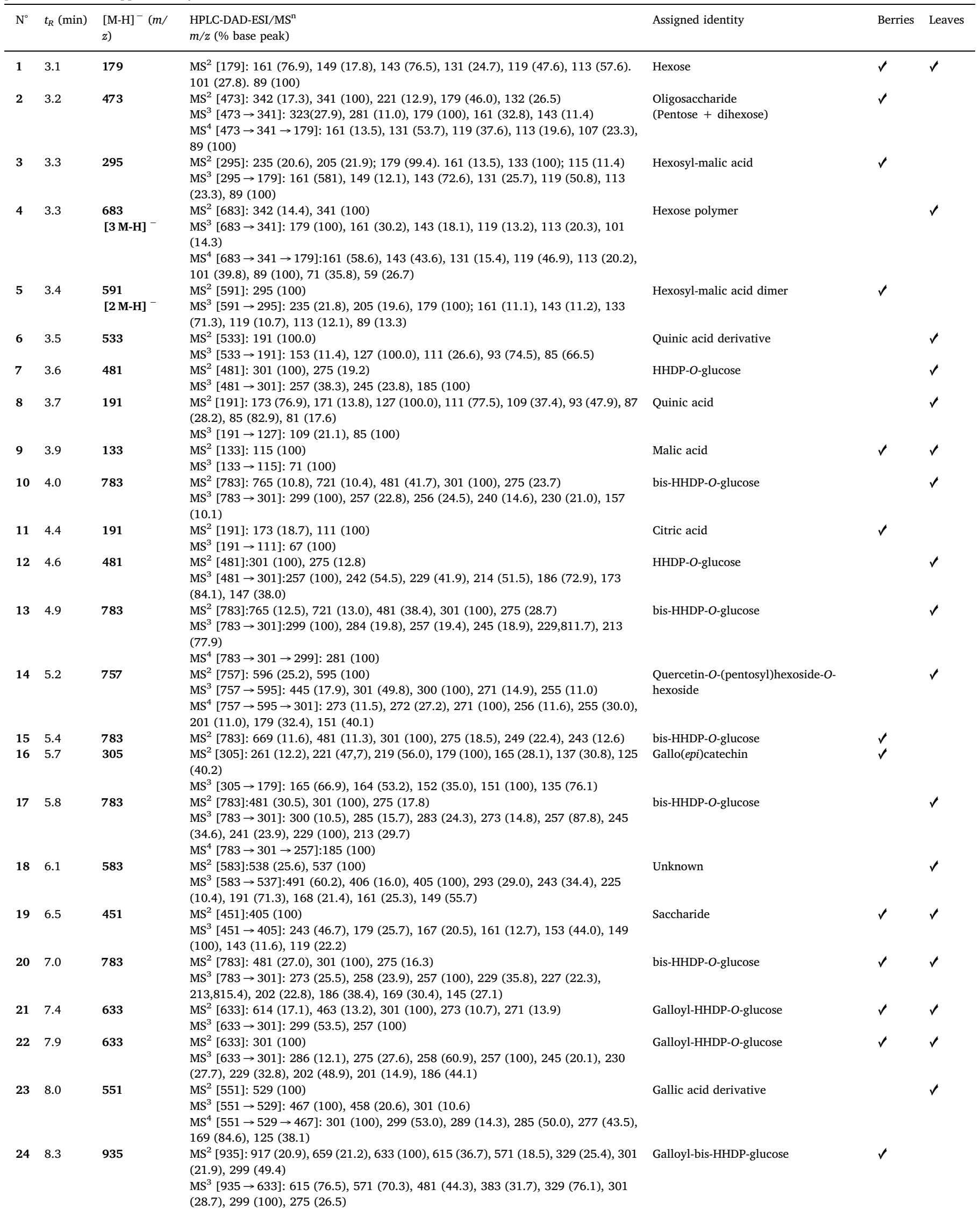


Table 1 (continued)

\begin{tabular}{|c|c|c|c|c|c|c|}
\hline $\mathrm{N}^{\circ}$ & $t_{R}(\min )$ & $\begin{array}{l}{[\mathrm{M}-\mathrm{H}]^{-}(\mathrm{m} /} \\
z)\end{array}$ & $\begin{array}{l}\text { HPLC-DAD-ESI/MS } \\
m / z \text { (\% base peak) }\end{array}$ & Assigned identity & Berries & Leaves \\
\hline 25 & 8.5 & 563 & $\begin{array}{l}\operatorname{MS}^{2}[563]: 517(100) \\
\operatorname{MS}^{3}[563 \rightarrow 517]: 385(93.1), 223(89.7), 208(100), 164(52.3) \\
M^{4}[563 \rightarrow 517 \rightarrow 208]: 164(100)\end{array}$ & $\begin{array}{l}\text { Sinapic acid-O-pentosyl(hexoside) } \\
\text { (formate adduct) }\end{array}$ & $\checkmark$ & \\
\hline 26 & 8.6 & 755 & $\begin{array}{l}\mathrm{MS}^{2}[755]: 610(23.7), 609(100) \\
\operatorname{MS}^{3}[755 \rightarrow 609]: 430(11.9), 429(57.4), 285(100), 284(55.4), 257(17.2), 255 \\
(15.2) \\
M^{4}[755 \rightarrow 609 \rightarrow 285]: 257(64.6), 255(79.8), 229(43.9), 164(18.6), 151(100)\end{array}$ & $\begin{array}{l}\text { Kaempferol-O-dihexoside- } O \text { - } \\
\text { rhamnoside }\end{array}$ & $\checkmark$ & \\
\hline 27 & 8.7 & 741 & $\begin{array}{l}\mathrm{MS}^{2} \text { [741]: } 595(100), 446(11.3), 271(10.6) \\
\mathrm{MS}^{3}[741 \rightarrow 595]: 475(33.1), 449(22.3), 301(44.4), 300(100), 271(15.5), 215 \\
(11.0), 179(11.1) \\
\mathrm{MS}^{4}[741 \rightarrow 595 \rightarrow 300]: 271(22.7), 255(42.5), 179(100)\end{array}$ & $\begin{array}{l}\text { Quercetin-O-(pentosyl)hexoside- } O \text { - } \\
\text { rhamnoside }\end{array}$ & $\checkmark$ & $\checkmark$ \\
\hline 28 & 8.8 & 563 & $\begin{array}{l}\text { MS2 [563]: } 518(24.4), 517(100) \\
\text { MS3 [563 } \rightarrow 517]: 387(25.0), 385 \text { (90.7), } 365 \text { (45.5), } 293 \text { (26.3), } 223 \text { (90.1), } 208 \\
(100), 164(46.1)\end{array}$ & $\begin{array}{l}\text { Sinapic acid-O-(pentosyl)hexoside } \\
\text { (formate adduct) }\end{array}$ & & $\checkmark$ \\
\hline 29 & 9.0 & 385 & $\begin{array}{l}\mathrm{MS}^{2}[385]: 223(100), 205(73.6), 163(10.5) \\
\mathrm{MS}^{3}[385 \rightarrow 223]: 208(17.0), 164(100), 149(12.1)\end{array}$ & Sinapic acid- $O$-hexoside & $\checkmark$ & \\
\hline 30 & 9.1 & 741 & $\begin{array}{l}\mathrm{MS}^{2}[741]: 595(100), 446(12.0), 300(17.0) \\
\mathrm{MS}^{3}[741 \rightarrow 595]: 463(20.2), 445(43.7), 368(19.3), 343(36.2), 301(38.0), 300 \\
(100), 273(42.1), 257(10.1), 179(15.4) \\
\mathrm{MS}^{4}[741 \rightarrow 595 \rightarrow 300]: 271(100), 179(36.7)\end{array}$ & $\begin{array}{l}\text { Quercetin- } O \text {-(pentosyl)hexoside- } O \text { - } \\
\text { rhamnoside }\end{array}$ & $\checkmark$ & \\
\hline 31 & 9.3 & 565 & $\begin{array}{l}\mathrm{MS}^{2} \text { [565]: } 520 \text { (13.0), } 519 \text { (17.8), } 403 \text { (100), } 385 \text { (14.7), } 325 \text { (10.9), } 223 \text { (66.9), } 221 \\
(15.4), 179 \text { (16.6) } \\
\left.\mathrm{MS}^{3} \text { [565 } \rightarrow 403\right]: 223 \text { (89.5), } 149 \text { (27.5), } 135 \text { (100) }\end{array}$ & Caffeic acid-O-(sinapoyl-O-hexoside) & $\checkmark$ & $\checkmark$ \\
\hline 32 & 9.5 & 431 & $\begin{array}{l}\operatorname{MS}^{2}[431]: 385(100), 223(13.5) \\
\operatorname{MS}^{3}[431 \rightarrow 385]: 223(64.4), 206(11.7), 205(48.9), 161(21.2), 153(100), 138 \\
(18.5) \\
\operatorname{MS}^{4}[431 \rightarrow 385 \rightarrow 153]: 138(100), 136(56.9), 114(40.8), 97(26.7)\end{array}$ & Roseoside (formate adduct) & $\checkmark$ & $\checkmark$ \\
\hline 33 & 9.8 & 759 & $\begin{array}{l}\mathrm{MS}^{2}[759]: 651(16.0), 639(30.8), 621(20.3), 579(53.8), 549(100) \\
\mathrm{MS}^{3}[759 \rightarrow 549]: 531(21.1), 519(49.8), 491(100), 477(83.4), 315(52.0), 271 \\
(15.2) \\
\mathrm{MS}^{4}[759 \rightarrow 549 \rightarrow 491]: 315(100), 300(76.9), 299(40.8), 271(36.7)\end{array}$ & Isorhamnetin-O-glucuronide derivative & $\checkmark$ & \\
\hline 34 & 10.5 & 489 & $\begin{array}{l}\mathrm{MS}^{2} \text { [489]: } 446(10.6), 295(15.0), 283(11.9), 265(18.5), 223(100), 208(11.6), 205 \\
(45.3), 190(30.4), 175(20.7), 164(34.1), 149(37.5) \\
\left.M^{3} \text { [489 } \rightarrow 223\right]: 208(77.7), 179(100), 164(43.9)\end{array}$ & Sinapic acid derivative & & $\checkmark$ \\
\hline 35 & 10.6 & 385 & $\begin{array}{l}\operatorname{MS}^{2}[385]: 325(100), 295(92.3), 265(84.6), 223(25.8) \\
\operatorname{MS}^{3}[385 \rightarrow 325]: 307(34.3), 265(82.0), 223(100), 206(48.1) \\
M^{4}[385 \rightarrow 325 \rightarrow 223]: 164(100)\end{array}$ & Sinapic acid-O-hexoside & $\checkmark$ & $\checkmark$ \\
\hline 36 & 10.7 & 725 & $\begin{array}{l}\mathrm{MS}^{2}[725]: 622(12.8), 580(35.0), 579(100) \\
\mathrm{MS}^{3}[725 \rightarrow 579]: 521(22.7), 447(23.5), 429(33.9), 326(18.8), 285 \text { (100), } 257 \\
(18.7), 255(15.9)\end{array}$ & $\begin{array}{l}\text { Kaempferol-O-(pentosyl)hexoside- } O \text { - } \\
\text { rhamnoside }\end{array}$ & $\checkmark$ & \\
\hline 37 & 10.9 & 449 & $\begin{array}{l}\operatorname{MS}^{2}[449]: 287(100), 269(32.4), 259(53.9) \\
\text { MS }^{3}[449 \rightarrow 287]: 259(100), 243(27.0), 201(17.2), 173(20.6), 151(63.6), 125 \\
(40.1), 119(10.6)\end{array}$ & Dihydrokaempferol-O-hexoside & $\checkmark$ & \\
\hline 38 & 11.1 & 755 & $\begin{array}{l}\mathrm{MS}^{2}[755]: 609(100) \\
\mathrm{MS}^{3}[755 \rightarrow 609]: 459(25.7), 315(100), 300(13.4), 299 \text { (13.6), } 271(16.9), 243 \\
(13.3) \\
\mathrm{MS}^{4}[755 \rightarrow 609 \rightarrow 315]: 301(21.1), 300(100), 299(91.8), 298(16.1), 259(20.9)\end{array}$ & $\begin{array}{l}\text { Isorhamnetin-O-(pentosyl)hexoside- } O \text { - } \\
\text { rhamnoside }\end{array}$ & $\checkmark$ & \\
\hline 39 & 11.2 & 611 & $\begin{array}{l}\mathrm{MS}^{2}[611]: 474(15.9), 463(22.8), 447(17.0), 329(12.3), 317(100), 272(11.0), \\
270(20.0), 251(18.4) \\
\mathrm{MS}^{3}[611 \rightarrow 317]: 272(76.2), 271(100), 179(25.0)\end{array}$ & Myricetin-O-(pentosyl)hexoside & $\checkmark$ & \\
\hline 40 & 11.4 & 463 & $\begin{array}{l}\operatorname{MS}^{2}[463]: 301(100), 300(35.6) \\
\operatorname{MS}^{3}[463 \rightarrow 301]: 300 \text { (90.9), } 257 \text { (100), } 229 \text { (61.3), } 207 \text { (27.7), } 172(33.3)\end{array}$ & Ellagic acid-O-hexoside & & $\checkmark$ \\
\hline 41 & 11.8 & 503 & $\begin{array}{l}\mathrm{MS}^{2}[503]: 223(100), 205(56.7), 191(22.0), 164(14.1), 149(25.9) \\
\mathrm{MS}^{3}[503 \rightarrow 223]: 179(100), 164(65.5), 149(76.1)\end{array}$ & Sinapic acid derivative & & $\checkmark$ \\
\hline 42 & 12.7 & 475 & $\begin{array}{l}\operatorname{MS}^{2}[475]: 301(100), 275(11.3) \\
\left.\text { MS }^{3} \text { [475 } \rightarrow 301\right]: 257(100), 230(22.3), 213 \text { (27.5), } 201 \text { (29.8), } 200 \text { (29.0), } 192 \\
(34.7), 186(14.7), 185(34.0)\end{array}$ & Ellagic acid-O-(acetyl)pentoside & & $\checkmark$ \\
\hline 43 & 13.0 & 625 & $\begin{array}{l}\mathrm{MS}^{2} \text { [625]: } 505 \text { (15.6), } 463(12.0), 445 \text { (38.2), } 301 \text { (100), } 300 \text { (97.6), } 271 \text { (21.3), } 255 \\
(22.0) \\
\text { MS }^{3}[625 \rightarrow 301]: 271(100), 179 \text { (50.6), } 151 \text { (41.5) }\end{array}$ & Quercetin-O-dihexoside & & $\checkmark$ \\
\hline 44 & 13.2 & 371 & $\begin{array}{l}\operatorname{MS}^{2}[371]: 249 \text { (100) } \\
\left.\operatorname{MS}^{3} \text { [371 } \rightarrow 249\right]: 231 \text { (41.1), } 113 \text { (100), } 99 \text { (12.5), } 95 \text { (27.3), } 85 \text { (49.4) }\end{array}$ & Unknown & $\checkmark$ & $\checkmark$ \\
\hline 45 & 13.9 & 371 & $\begin{array}{l}\operatorname{MS}^{2}[371]: 249(100) \\
\operatorname{MS}^{3}[371 \rightarrow 249]: 231 \text { (47.1), } 175 \text { (10.9), } 113 \text { (100), } 111 \text { (12.2), } 99 \text { (20.5), } 85 \text { (36.3) }\end{array}$ & Unknown & & $\checkmark$ \\
\hline 46 & 14.0 & 389 & $\begin{array}{l}\operatorname{MS}^{2} \text { [389]: } 209(100), 181(19.1), 179(76.2), 135(35.8) \\
\operatorname{MS}^{3}[389 \rightarrow 209]: 135(100)\end{array}$ & Caffeic acid derivative & $\checkmark$ & \\
\hline 47 & 14.3 & 725 & $\begin{array}{l}\mathrm{MS}^{2}[725]: 545(100), 313(12.9) \\
\mathrm{MS}^{3}[725 \rightarrow 545]: 351(13.0), 313(100), 295(20.2), 249 \text { (11.4), } 247 \text { (12.5), } 231 \\
(26.8), 229(18.2), 187(16.5), 179(10.8), 161(13.9) \\
\text { MS }^{4}[725 \rightarrow 545 \rightarrow 313]: 179(100), 161(85.9), 115(40.7), 113(63.4), 101(63.4), \\
89(49.0)\end{array}$ & Saccharide & & $\checkmark$ \\
\hline 48 & 14.4 & 595 & $\begin{array}{l}\mathrm{MS}^{2}[595]: 445(14.8), 301(46.2), 300(100), 271(20.6) \\
\operatorname{MS}^{3}[595 \rightarrow 301]: 271(100), 257(12.2), 255(89.0), 243(10.0), 179(42.8), 151\end{array}$ & Quercetin-O-(pentosyl)hexoside & $\checkmark$ & $\checkmark$ \\
\hline
\end{tabular}


Table 1 (continued)

\begin{tabular}{|c|c|c|c|c|c|c|}
\hline $\mathrm{N}^{\circ}$ & $t_{R}(\min )$ & $\begin{array}{l}{[\mathrm{M}-\mathrm{H}]^{-}(\mathrm{m} /} \\
z)\end{array}$ & $\begin{array}{l}\text { HPLC-DAD-ESI/MS } \\
m / z(\% \text { base peak })\end{array}$ & Assigned identity & Berries & Leaves \\
\hline 49 & 14.8 & 433 & $\begin{array}{l}\operatorname{MS}^{2}[433]: 301(100), 300(52.3 \\
\operatorname{MS}^{3}[433 \rightarrow 301]: 300(100), 257 \text { (40.7), } 179(53.8), 151(55.5)\end{array}$ & Quercetin- $O$-pentoside & $\checkmark$ & \\
\hline 50 & 15.1 & 609 & $\begin{array}{l}\mathrm{MS}^{2}[609]: 576(57.3), 447(72.2), 301(48.2), 285(100), 255(25.1) \\
\operatorname{MS}^{3}[609 \rightarrow 285]: 255(100)\end{array}$ & Kaempferol-O-dihexoside & $\checkmark$ & \\
\hline 51 & 15.1 & 595 & $\begin{array}{l}\mathrm{MS}^{2}[595]: 445 \text { (10.9), } 301(57.6), 300(100), 271(15.4), 255(11.9) \\
\operatorname{MS}^{3}[595 \rightarrow 300]: 271(100), 255(59.9), 179(36.9), 151(34.4)\end{array}$ & Quercetin-O-pentosyl(hexoside) & & $\checkmark$ \\
\hline 52 & 15.5 & 547 & $\begin{array}{l}\text { MS }^{2}[547]: 503(10.6), 311(100), 265(10.9), 221(13.6) \\
\text { MS }^{3}[547 \rightarrow 311]: 293(23.9), 275(36.0), 251(62.5), 233(50.0), 221(22.0), 179 \\
(29.5), 161(16.7), 149(100), 113(46.6)\end{array}$ & Saccharide & $\checkmark$ & \\
\hline 53 & 15.7 & 551 & $\begin{array}{l}\operatorname{MS}^{2}[551]: 505(100) \\
\operatorname{MS}^{3}[551 \rightarrow 505]: 373(100), 179(22.3), 161(54.4) \\
\operatorname{MS}^{4}[551 \rightarrow 505 \rightarrow 373]: 161(100), 119(11.6), 113(15.9)\end{array}$ & Saccharide & & $\checkmark$ \\
\hline 54 & 16.1 & 639 & $\begin{array}{l}\text { MS }^{2} \text { [639]: } 477(21.0), 315(100), 300(22.6), 299(17.3), 271(16.2) \\
\operatorname{MS}^{3}[639 \rightarrow 315]: 301(11.0), 300(100), 299(22.6)\end{array}$ & Isorhamnetin-O-dihexoside & & $\checkmark$ \\
\hline 55 & 16.3 & 547 & $\begin{array}{l}\operatorname{MS}^{2}[547]: 311(100), 191(14.1), 161(21.8) \\
\text { MS }^{3}[547 \rightarrow 311]: 293(15.1), 251(22.0), 179(42.4), 161 \text { (78.9), } 149 \text { (100), } 119 \\
(10.3), 101(85.1)\end{array}$ & Saccharide & & $\checkmark$ \\
\hline 56 & 16.6 & 597 & $\begin{array}{l}\mathrm{MS}^{2}[597]: 489(15.1), 477(49.4), 459(17.2), 417(15.9), 387(67.8), 357(100) \\
\mathrm{MS}^{3}[597 \rightarrow 357]: 209(100), 139(11.0), 123(27.6) \\
\mathrm{MS}^{3}[597 \rightarrow 387]: 315(100), 239(37.1), 221(14.8), 191(18.5), 167(12.0), 161 \\
(11.0), 153(10.4) \\
\mathrm{MS}^{4}[597 \rightarrow 357 \rightarrow 209]: 165(28.3), 164(63.7), 123(100), 121(23.6) \\
\mathrm{MS}^{4}[597 \rightarrow 357 \rightarrow 315]: 209(41.4), 190(21.3), 167(100), 139(28.5) 126(37.7)\end{array}$ & Unknown & $\checkmark$ & \\
\hline 57 & 17.1 & 551 & $\begin{array}{l}\operatorname{MS}^{2}[551]: 505(100) \\
\operatorname{MS}^{3}[551 \rightarrow 505]: 373(100), 161(22.7) \\
\operatorname{MS}^{4}[551 \rightarrow 505 \rightarrow 373]: 179(17.9), 161(100), 143(18.3), 113(25.9)\end{array}$ & Saccharide & & $\checkmark$ \\
\hline 58 & 17.4 & 547 & $\begin{array}{l}\operatorname{MS}^{2}[547]: 311(100), 179(14.1), 161(21.8) \\
\operatorname{MS}^{3}[547 \rightarrow 311]: 293(15.1), 251(22.0), 179(42.4), 161 \text { (78.9), } 149 \text { (100), } 119 \\
(10.3), 101(85.1)\end{array}$ & Saccharide & & $\checkmark$ \\
\hline 59 & 18.0 & 415 & $\begin{array}{l}\mathrm{MS}^{2} \text { [415]: } 370 \text { (51.9), } 227 \text { (75.2), } 225 \text { (81.0), } 187 \text { (67.1), } 179 \text { (100), } 161 \text { (23.9), } 131 \\
(14.5) \\
\left.\text { MS }^{3} \text { [415 } \rightarrow 179\right]: 161 \text { (100), } 143 \text { (14.2), } 119 \text { (15.3) }\end{array}$ & Saccharide & $\checkmark$ & \\
\hline 60 & 18.9 & 609 & $\begin{array}{l}\mathrm{MS}^{2}[609]: 578(10.7), 477(43.7), 357(11.1), 315(100), 314(82.8), 300(29.7) \\
\text { MS }^{3}[609 \rightarrow 315]: 301(20.6), 300(100), 299(72.2), 287(10.1), 271(58.1), 255 \\
(39.7), 243(39.6)\end{array}$ & Isorhamnetin- $O$-(pentosyl)hexoside & $\checkmark$ & \\
\hline 61 & 19.0 & 463 & $\begin{array}{l}\operatorname{MS}^{2}[463]: 415(99.4), 301(100), 299(37.1) \\
\operatorname{MS}^{3}[463 \rightarrow 301]: 151(100)\end{array}$ & Quercetin-O-hexoside & $\checkmark$ & \\
\hline 62 & 19.0 & 549 & $\begin{array}{l}\operatorname{MS}^{2}[549]: 503(100) \\
\operatorname{MS}^{3}[549 \rightarrow 503]: 417(10.0), 371(100), 353(11.6), 191(14.3), 173(10.1), 161 \\
(37.5), 149(25.5), 143(10.5) \\
\operatorname{MS}^{4}[549 \rightarrow 503 \rightarrow 371]: 161(100)\end{array}$ & Saccharide & & $\checkmark$ \\
\hline 63 & 19.5 & 579 & $\begin{array}{l}\operatorname{MS}^{2}[579]: 429(28.1), 339(10.6), 285(100), 284(47.0), 257(12.3), 255(14.9) \\
\operatorname{MS}^{3}[579 \rightarrow 285]: 257(94.1), 255(100), 229(83.2), 199(28.9), 151(54.8)\end{array}$ & Kaempferol-O-pentosyl(hexoside) & & $\checkmark$ \\
\hline 64 & 19.6 & 549 & $\begin{array}{l}\operatorname{MS}^{2}[549]: 504(16.9), 503(100) \\
\text { MS }^{3}[549 \rightarrow 503]: 372(28.9), 371(100), 293(17.3), 179(15.4), 161(22.3) \\
\text { MS }^{4}[549 \rightarrow 503 \rightarrow 371]: 179(49.8), 161(100), 131(15.8), 119(23.3), 113(55.1)\end{array}$ & Saccharide (formate adduct) & & $\checkmark$ \\
\hline 65 & 20.4 & 609 & $\begin{array}{l}\text { MS }^{2}[609]: 577(10.7), 459(35.1), 315(100), 300(25.9), 299(21.1), 271(14.8) \\
\text { MS }^{3}[609 \rightarrow 315]: 300(100), 299(72.2), 271(45.8), 255(45.9), 243(11.5)\end{array}$ & Isorhamnetin- $O$-pentosyl(hexoside) & & $\checkmark$ \\
\hline 66 & 20.6 & 483 & $\begin{array}{l}\mathrm{MS}^{2}[483]: 434(15.8), 413(21.6), 410(31.9), 331(50.3), 313 \text { (32.7), } 211 \text { (90.9), } \\
177 \text { (75.7), } 169(100), 151(24.1) \\
\mathrm{MS}^{3}[483 \rightarrow 169]: 125(100)\end{array}$ & Digalloyl-O-glucoside & & $\checkmark$ \\
\hline 67 & 21.3 & 839 & $\begin{array}{l}\mathrm{MS}^{2}[839]: 639(14.4), 625(100), 300(11.5) \\
\mathrm{MS}^{3}[839 \rightarrow 625]: 505(26.6), 463(11.8), 445(67.8), 301(55.2), 300(100), 299 \\
(11.1), 271(45.6), 179(22.3) \\
\mathrm{MS}^{4}[839 \rightarrow 625 \rightarrow 301]: 271(100), 257(11.4), 179(66.0), 151(26.6)\end{array}$ & Quercetin-O-dihexoside derivative & & $\checkmark$ \\
\hline 68 & 24.5 & 689 & $\begin{array}{l}\operatorname{MS}^{2}[689]: 635(57.5), 519(27.4), 465(100), 313(21.5) \\
\text { MS }^{3}[689 \rightarrow 465]: 313(100), 295(25.8), 169(12.2), 125(19.8) \\
\text { MS }^{4}[689 \rightarrow 465 \rightarrow 313]: 169(100), 125(21.5)\end{array}$ & Trigalloylglucose derivative & & $\checkmark$ \\
\hline 69 & 24.7 & 447 & $\begin{array}{l}\mathrm{MS}^{2}[447]: 285(59.3), 284(100), 255(26.5) \\
\operatorname{MS}^{3}[447 \rightarrow 285]: 255(100), 229(21.4)\end{array}$ & Kaempferol-O-hexoside & & $\checkmark$ \\
\hline 70 & 25.8 & 515 & $\begin{array}{l}\operatorname{MS}^{2}[515]: 353(100) \\
\operatorname{MS}^{3}[515 \rightarrow 353]: 191 \text { (100), } 179 \text { (29.3), } 161(10.8), 135 \text { (15.7) }\end{array}$ & 3,5-O-Dicaffeoylquinic acid & $\checkmark$ & \\
\hline 71 & 25.7 & 649 & $\begin{array}{l}\operatorname{MS}^{2}[649]: 469(100) \\
\operatorname{MS}^{3}[649 \rightarrow 469]: 425(27.8), 237(18.0), 231(12.7), 205(37.0), 187(79.7), 161 \\
(100) \\
\operatorname{MS}^{4}[649 \rightarrow 469 \rightarrow 161]: 129(100) \\
\operatorname{MS}^{4}[649 \rightarrow 469 \rightarrow 187]: 143(100)\end{array}$ & 2-Methylaconitate derivative & & $\checkmark$ \\
\hline 72 & 25.8 & 477 & $\begin{array}{l}\mathrm{MS}^{2} \text { [477]: } 467 \text { (20.9) } 358(26.0), 315 \text { (100), } 301 \text { (14.9), } 287 \text { (42.3), } 257 \text { (10.9), } 244 \\
(14.0), 173 \text { (42.9) } \\
\left.\mathrm{MS}^{3} \text { [477 } \rightarrow 315\right]: 300 \text { (100), } 285 \text { (17.1), } 271(31.9)\end{array}$ & Isorhamnetin- $O$-hexoside & $\checkmark$ & \\
\hline 73 & 26.1 & 447 & $\begin{array}{l}\mathrm{MS}^{2}[447]: 327(10.2), 285(100), 255(16.2) \\
\mathrm{MS}^{3}[447 \rightarrow 285]: 255 \text { (100), } 229(10.9), 201(25.3)\end{array}$ & Kaempferol-O-hexoside & $\checkmark$ & $\checkmark$ \\
\hline 74 & 26.9 & 477 & & Isorhamnetin- $O$-hexoside & $\checkmark$ & $\checkmark$ \\
\hline
\end{tabular}


Table 1 (continued)

\begin{tabular}{|c|c|c|c|c|c|c|}
\hline $\mathrm{N}^{\circ}$ & $t_{R}(\min )$ & $\begin{array}{l}{[\mathrm{M}-\mathrm{H}]^{-}(m /} \\
z)\end{array}$ & $\begin{array}{l}\text { HPLC-DAD-ESI/MS } \\
m / z \text { (\% base peak) }\end{array}$ & Assigned identity & Berries & Leaves \\
\hline 75 & 27.0 & 845 & $\begin{array}{l}\mathrm{MS}^{2} \text { [477]: } 357(10.3), 315(100), 301(10.5), 285(27.4), 271(14.1) \\
\mathrm{MS}^{3}[477 \rightarrow 315]: 301(51.7), 300(22.2), 299(76.9), 286(86.6), 285(54.7), 271 \\
(100), 257(52.6), 243(23.3) \\
\mathrm{MS}^{2} \text { [845]: } 653(82.1), 639(100), 515(18.0), 459(12.8), 413(11.1), 330(11.0), 315 \\
(23.0), 300(10.4) \\
\mathrm{MS}^{3}[845 \rightarrow 639]: 607(12.6), 491(21.9), 477(14.4), 459(40.4), 417(18.4), 393 \\
(21.1), 315(100), 300(41.9) \\
\mathrm{MS}^{4}[845 \rightarrow 639 \rightarrow 315]: 301(24.4), 300(100), 299(49.6), 257(13.2)\end{array}$ & Isorhamnetin-O-(sinapoyl)dihexoside & & $\checkmark$ \\
\hline 76 & 27.1 & 447 & $\begin{array}{l}\mathrm{MS}^{2}[447]: 285(100), 284(97.4), 255(29.8) \\
\mathrm{MS}^{3}[447 \rightarrow 285]: 255(100), 229(10.3)\end{array}$ & Kaempferol-O-hexoside & & $\checkmark$ \\
\hline 77 & 27.9 & 579 & $\begin{array}{l}\operatorname{MS}^{2}[579]: 533(14.3), 315(100), 299(13.4), 271(14.8) \\
\operatorname{MS}^{3}[579 \rightarrow 315]: 300(100), 299(15.1), 271(34.9), 151(15.6)\end{array}$ & Isorhamnetin-O-dipentoside & & $\checkmark$ \\
\hline 78 & 28.0 & 429 & $\operatorname{MS}^{2}$ [429]: 249 (100), 205 (89.2), 179 (13.7), 161 (63.7), 135 (17.2) & Caffeic acid derivative & & $\checkmark$ \\
\hline 79 & 28.7 & 815 & $\begin{array}{l}\mathrm{MS}^{2}[815]: 653(63.0), 639(100), 485(11.4), 329(11.0), 315(18.9) \\
\mathrm{MS}^{3}[815 \rightarrow 639]: 607(18.8), 580(23.4), 491(20.7), 459(46.1), 433(18.3), 357 \\
(53.8), 315(100), 301(25.7), 300(46.2), 299(16.1), 271(26.5) \\
\mathrm{MS}^{4}[815 \rightarrow 639 \rightarrow 315]: 300(100), 299(42.9), 285(16.3)\end{array}$ & $\begin{array}{l}\text { Isorhamnetin- } O \text {-dihexoside- } O \text { - } \\
\text { glucuronide }\end{array}$ & & $\checkmark$ \\
\hline 80 & 29.3 & 755 & $\begin{array}{l}\mathrm{MS}^{2}[755]: 609(100) \\
\mathrm{MS}^{3}[755 \rightarrow 609]: 429(57.5), 285(100), 284(33.9), 255(11.2) \\
\mathrm{MS}^{4}[755 \rightarrow 609 \rightarrow 285]: 257(25.0), 255(100)\end{array}$ & Kaempferol-O-(coumaroyl)dihexoside & $\checkmark$ & \\
\hline 81 & 30.5 & 711 & $\begin{array}{l}\mathrm{MS}^{2}[711]: 665(10.5), 505(10.2), 503(100) \\
\mathrm{MS}^{3}[711 \rightarrow 503]: 485(55.0), 453(100), 421(78.3), 417(61.3), 410 \text { (53.2), } 409 \\
(92.0), 380(50.4) \\
\text { MS }^{4}[711 \rightarrow 503 \rightarrow 453]: 409(100)\end{array}$ & $\begin{array}{l}\text { Triterpene acid- } O \text {-hexoside (formate } \\
\text { adduct) }\end{array}$ & $\checkmark$ & $\checkmark$ \\
\hline 82 & 31.1 & 727 & $\begin{array}{l}\operatorname{MS}^{2}[727]: 681(100), 619(27.7) \\
\operatorname{MS}^{3}[727 \rightarrow 681]: 619(100) \\
\operatorname{MS}^{4}[727 \rightarrow 681 \rightarrow 619]: 457(100), 425(25.3)\end{array}$ & Unknown & & $\checkmark$ \\
\hline 83 & 31.9 & 711 & $\begin{array}{l}\operatorname{MS}^{2}[711]: 665(100) \\
\operatorname{MS}^{3}[711 \rightarrow 665]: 621(100), 589(27.5), 459(13.4) \\
\operatorname{MS}^{3}[711 \rightarrow 665 \rightarrow 621]: 590(100), 459(26.7), 428(54.5) ; 459(100)\end{array}$ & Unknown & & $\checkmark$ \\
\hline 84 & 32.7 & 805 & $\begin{array}{l}\mathrm{MS}^{2}[805]: 639(100), 459(30.1), 315(87.2), 300(26.0), 271(11.4) \\
\mathrm{MS}^{3}[805 \rightarrow 315]: 300 \text { (100), } 299(33.8), 287(17.9), 151(23.0)\end{array}$ & Isorhamnetin-O-dihexoside derivative & & $\checkmark$ \\
\hline 85 & 33.0 & 591 & $\begin{array}{l}\mathrm{MS}^{2} \text { [591]:567 (52.0), } 544(30.7), 367(24.9), 265(28.4,223(100), 205(28.1), 190 \\
(14.9), 164(12.8) \\
\mathrm{MS}^{3}[591 \rightarrow 223]: 208(88.8), 179(17.0), 164(100)\end{array}$ & Disinapoyl-O-hexoside & & $\checkmark$ \\
\hline 86 & 34.9 & 581 & $\begin{array}{l}\mathrm{MS}^{2}[581]: 559(100), 558(31.9) \\
\mathrm{MS}^{3}[581 \rightarrow 559]: 535(21.4), 477(10.4), 455(20.2), 454(100) \\
\mathrm{MS}^{4}[581 \rightarrow 559 \rightarrow 454]: 373(100)\end{array}$ & Unknown & & $\checkmark$ \\
\hline 87 & 35.8 & 593 & $\begin{array}{l}\mathrm{MS}^{2}[593]: 447(12.1), 284(100) \\
\mathrm{MS}^{3}[593 \rightarrow 285]: 267(19.1), 257(56.0), 255(32.7), 229(31.2), 213 \text { (28.1), } 163 \\
(20.6), 151(100)\end{array}$ & Kaempferol-O-(coumaroyl)hexoside & $\checkmark$ & $\checkmark$ \\
\hline 88 & 36.2 & 581 & $\begin{array}{l}\operatorname{MS}^{2}[581]: 461(100), 341(29.4) \\
\operatorname{MS}^{3}[581 \rightarrow 461]: 341(100) \\
\operatorname{MS}^{4}[581 \rightarrow 461 \rightarrow 341]: 299(100), 284(37.4), 271(26.0), 151(44.6)\end{array}$ & Diosmetin-8- $C$-hexoside- $C$-hexoside & $\checkmark$ & \\
\hline 89 & 36.6 & 593 & $\begin{array}{l}\mathrm{MS}^{2}[593]: 447(10.5), 286(12.5), 285(100) \\
\mathrm{MS}^{3}[593 \rightarrow 285]: 257(35.5), 241(24.1), 229 \text { (18.9), } 213(18.3), 151 \text { (1000), } 123 \\
(11.9)\end{array}$ & Kaempferol-O-(coumaroyl)hexoside & $\checkmark$ & $\checkmark$ \\
\hline 90 & 36.8 & 623 & $\begin{array}{l}\mathrm{MS}^{2}[623]: 461(78.3), 323(56.9), 315(11.0), 301(15.8), 299(100), 285(98.4), 256 \\
(21.4) \\
\operatorname{MS}^{3}[623 \rightarrow 299]: 284(100), 271(40.4), 256(47.3) \\
\operatorname{MS}^{4}[623 \rightarrow 299 \rightarrow 284]: 256(100), 151(43.1)\end{array}$ & Diosmetin-O-dihexoside & $\checkmark$ & \\
\hline 91 & 37.4 & 581 & $\begin{array}{l}\operatorname{MS}^{2}[581]: 461(100), 341(28.5) \\
\operatorname{MS}^{3}[581 \rightarrow 461]: 341(100) \\
\operatorname{MS}^{4}[581 \rightarrow 461 \rightarrow 341]: 299(100), 284(17.6), 151(68.8)\end{array}$ & Diosmetin-8-C-hexoside- $C$-hexoside & $\checkmark$ & \\
\hline 92 & 37.5 & 613 & $\begin{array}{l}\mathrm{MS}^{2}[613]: 492(100), 476(39.9), 466(40.0), 462(20.8), 342(10.3) \\
\mathrm{MS}^{3}[613 \rightarrow 492]: 451(16.5), 449(100), 373(22.8), 343(47.3), 342(62.6), 299 \\
(14.3), 160(11.7) \\
\mathrm{MS}^{4}[613 \rightarrow 492 \rightarrow 449]: 303(23.4), 299(100), 149(55.5), 145(17.2)\end{array}$ & Unknown & $\checkmark$ & \\
\hline 93 & 38.3 & 613 & $\begin{array}{l}\mathrm{MS}^{2}[613]: 492(100), 476(39.9), 466(40.0), 462(20.8), 342(10.3) \\
\mathrm{MS}^{3}[613 \rightarrow 492]: 451(16.5), 449(100), 373(22.8), 357(12.3), 343(47.3), 342 \\
(62.6), 299(14.3), 288(14.6), 145(10.3) \\
\mathrm{MS}^{4}[613 \rightarrow 492 \rightarrow 372]: 357(100), 175(51.1) \\
\mathrm{MS}^{4}[613 \rightarrow 492 \rightarrow 449]: 376(23.8), 329(25.8), 314(49.6), 303(23.0), 299(21.5), \\
289(14.1), 157(100), 149(14.1), 135(16.8) \\
\operatorname{MS}^{4}[612 \rightarrow 492 \rightarrow 449]: 275(100), 233(36.6), 174(88.5), 145(41.7), 134(20.2)\end{array}$ & Unknown & $\checkmark$ & \\
\hline 94 & 39.0 & 533 & $\begin{array}{l}\operatorname{MS}^{2}[533]: 487(100) \\
\operatorname{MS}^{3}[533 \rightarrow 487]: 451(24.4), 427(53.3), 355(46.0), 221(51.3), 191(45.1), 161 \\
(41.7), 149(100), 143(52.8) \\
\operatorname{MS}^{4}[533 \rightarrow 487 \rightarrow 149]: 131(12.4), 85(100)\end{array}$ & Saccharide & & $\checkmark$ \\
\hline
\end{tabular}

HHDP: Hexahydroxydiphenoyl. 
Table 2

Quantification (mg g ${ }^{-1}$ dry extract, DE) of main phenolic compounds of $E$. umbellata methanolic extracts (berries and leaves) before (Undigested) and after the complete in vitro gastrointestinal digestion (Digested). Data represent the mean \pm standard deviation $(n=3)$.

\begin{tabular}{|c|c|c|c|c|c|c|}
\hline \multirow[t]{2}{*}{$\mathrm{N}^{\circ}$} & \multirow[t]{2}{*}[\mathrm{M}-\mathrm{H}]{$^{-}$} & \multirow[t]{2}{*}{ Assigned identification } & \multicolumn{2}{|l|}{ Berries } & \multicolumn{2}{|l|}{ Leaves } \\
\hline & & & Undigested & Digested & Undigested & Digested \\
\hline \multicolumn{7}{|c|}{ Hydroxycinnamic acids } \\
\hline 28 & 565 & Sinapic acid-O-(pentosyl)hexoside & $0.10 \pm 0.01^{\mathrm{a}}$ & & $1.42 \pm 0.04^{\mathrm{b}}$ & N.D. \\
\hline 35 & 385 & Sinapic acid- $O$-hexoside & & & $0.18 \pm 0.02^{\mathrm{b}}$ & $0.08 \pm 0.01^{\mathrm{a}}$ \\
\hline 41 & 503 & Sinapic acid derivative & & & $0.31 \pm 0.01^{\mathrm{b}}$ & $0.11 \pm 0.01^{\mathrm{a}}$ \\
\hline 78 & 429 & Caffeic acid derivative & & & & \\
\hline 85 & 591 & Disinapoyl-O-hexoside & & & $0.21 \pm 0.02$ & N.D. \\
\hline Total & & & $0.10 \pm 0.01^{\mathrm{a}}$ & & $2.13 \pm 0.07^{\mathrm{c}}$ & $0.20 \pm 0.01^{\mathrm{b}}$ \\
\hline \multicolumn{7}{|c|}{ Flavonols } \\
\hline 14 & 757 & Quercetin-O-(pentosyl)dihexoside & & & $0.67 \pm 0.03^{\mathrm{b}}$ & $0.22 \pm 0.08^{\mathrm{a}}$ \\
\hline 26 & 755 & Kaempferol-O-dihexoside-O-rhamnoside & $0.06 \pm 0.01$ & & & \\
\hline 33 & 759 & Isorhamnetin-O-glucuronide derivative & $0.17 \pm 0.01$ & & & \\
\hline 37 & 449 & Dihydrokaempferol-O-hexoside & $0.21 \pm 0.01$ & & & \\
\hline 38 & 755 & Isorhamnetin- $O$-(pentosyl)hexoside-O-rhamnoside & $0.17 \pm 0.01$ & & & \\
\hline 43 & 625 & Quercetin-O-dihexoside & & & $0.59 \pm 0.02^{b}$ & $0.20 \pm 0.01^{\mathrm{a}}$ \\
\hline 48 & 595 & Quercetin-O-(pentosyl)hexoside & $0.19 \pm 0.01$ & & & \\
\hline 49 & 433 & Quercetin- $O$-pentoside & $0.47 \pm 0.01^{\mathrm{b}}$ & $0.19 \pm 0.05^{\mathrm{a}}$ & & \\
\hline 51 & 595 & Quercetin- $O$-pentosyl(hexoside) & & & $5.05 \pm 0.25^{\mathrm{b}}$ & $2.37 \pm 0.05^{\mathrm{a}}$ \\
\hline 54 & 639 & Isorhamnetin-O-dihexoside & & & $0.39 \pm 0.01^{\mathrm{b}}$ & $0.12 \pm 0.01^{\mathrm{a}}$ \\
\hline 60 & 609 & Isorhamnetin-O-(pentosyl)hexoside & $0.14 \pm 0.01$ & & & \\
\hline 61 & 463 & Quercetin-O-hexoside & $0.13 \pm 0.01$ & & & \\
\hline 65 & 609 & Isorhamnetin-O-pentosyl(hexoside) & & & $3.94 \pm 0.14^{\mathrm{b}}$ & $1.27 \pm 0.03^{\mathrm{a}}$ \\
\hline 73 & 447 & Kaempferol-O-hexoside & $0.34 \pm 0.01^{\mathrm{c}}$ & $0.13 \pm 0.02^{\mathrm{a}}$ & $0.51 \pm 0.02^{\mathrm{d}}$ & $0.22 \pm 0.01^{\mathrm{b}}$ \\
\hline 74 & 477 & Isorhamnetin-O-hexoside & & & $1.71 \pm 0.05^{\mathrm{b}}$ & $0.73 \pm 0.03^{\mathrm{a}}$ \\
\hline 79 & 815 & Isorhamnetin-O-dihexoside-O-glucuronide & & & & \\
\hline 80 & 755 & Kaempferol-O-(coumaroyl)dihexoside & $0.07 \pm 0.01$ & & & \\
\hline 84 & 805 & Isorhamnetin-O-dihexoside derivative & & & $0.94 \pm 0.01^{\mathrm{b}}$ & $0.22 \pm 0.01^{\mathrm{a}}$ \\
\hline 87 & 593 & Kaempferol-O-(coumaroyl)hexoside & $2.07 \pm 0.13^{c}$ & $0.98 \pm 0.03^{\mathrm{a}}$ & $2.02 \pm 0.05^{\mathrm{c}}$ & $1.17 \pm 0.31^{\mathrm{b}}$ \\
\hline 89 & 593 & Kaempferol-O-(coumaroyl)hexoside & $0.37 \pm 0.05^{\mathrm{c}}$ & $0.14 \pm 0.01^{\mathrm{a}}$ & $0.62 \pm 0.02^{\mathrm{d}}$ & $0.21 \pm 0.02^{\mathrm{b}}$ \\
\hline Total & & & $4.38 \pm 0.12^{\mathrm{b}}$ & $1.43 \pm 0.08^{\mathrm{a}}$ & $16.45 \pm 0.55^{\mathrm{d}}$ & $6.74 \pm 0.43^{\mathrm{c}}$ \\
\hline \multicolumn{7}{|c|}{ Flavones } \\
\hline 88 & 583 & Diosmetin-8-C-hexoside- $C$-hexoside & $0.09 \pm 0.01$ & & & \\
\hline 90 & 623 & Diosmetin-O-dihexoside & $0.1 \pm 0.01^{\mathrm{b}}$ & $0.06 \pm 0.01^{\mathrm{a}}$ & & \\
\hline 91 & 583 & Diosmetin-8- $C$-hexoside- $C$-hexoside & $0.24 \pm 0.01^{\mathrm{b}}$ & $0.11 \pm 0.01^{\mathrm{a}}$ & & \\
\hline Total & & & $0.43 \pm 0.03^{b}$ & $0.18 \pm 0.02^{\mathrm{a}}$ & & \\
\hline \multicolumn{7}{|c|}{ Flavan-3-ols } \\
\hline 16 & 305 & Gallo(epi)catechin & $0.15 \pm 0.01^{\mathrm{a}}$ & & $0.85 \pm 0.03^{\mathrm{b}}$ & \\
\hline Total & & & $0.15 \pm 0.01^{\mathrm{a}}$ & & $0.85 \pm 0.03^{b}$ & \\
\hline \multicolumn{7}{|c|}{ Ellagic acid derivatives/ Ellagitannins } \\
\hline 13 & 783 & bis-HHDP-O-glucose & & & $1.21 \pm 0.03$ & \\
\hline 15 & 783 & bis-HHDP-O-glucose & & & $3.28 \pm 0.09^{\mathrm{b}}$ & $1.54 \pm 0.13^{\mathrm{a}}$ \\
\hline 17 & 783 & bis-HHDP-O-glucose & & & $10.77 \pm 0.13^{\mathrm{b}}$ & $4.03 \pm 0.32^{\mathrm{a}}$ \\
\hline 21 & 633 & Galloyl-HHDP-O-hexoside & $0.16 \pm 0.01$ & & $6.25 \pm 0.04^{\mathrm{b}}$ & $2.39 \pm 0.09^{\mathrm{a}}$ \\
\hline 24 & 935 & Galloyl-bis-HHDP-O-glucose & $0.35 \pm 0.01$ & & & \\
\hline 40 & 463 & Ellagic acid-O-hexoside & & & $0.28 \pm 0.01$ & N.D. \\
\hline 42 & 475 & Ellagic acid-O-(acetyl)pentoside & & & $1.15 \pm 0.01^{\mathrm{b}}$ & $0.61 \pm 0.03^{\mathrm{a}}$ \\
\hline Total & & & $0.50 \pm 0.03^{a}$ & & $22.93 \pm 0.30^{c}$ & $8.58 \pm 0.33^{b}$ \\
\hline TIPC & & & $5.56 \pm 0.19^{b}$ & $1.61 \pm 0.09^{\mathrm{a}}$ & $42.35 \pm 0.95^{\mathrm{d}}$ & $15.52 \pm 0.84^{\circ}$ \\
\hline
\end{tabular}

N.D.: not determined. HHDP: Hexahydroxydiphenoyl; TIPC: Total individual phenolic content. Bold values represent the sum of each type of components. Means not sharing the same letter are significantly different at $p<.05$ probality level.

\subsection{In vitro inhibition of digestive enzymes}

Inhibition of dietary sugar and fat intestinal metabolism seems to be an effective way to prevent T2DM and obesity (Grussu, Stewart, \& McDougall, 2011; McDougall, Kulkarni, \& Stewart, 2009; Podsędek et al., 2014; You et al., 2011). Currently, acarbose, miglitol, voglibose and orlistat are approved oral drugs for this purpose (Grussu et al., 2011; McDougall \& Stewart, 2005). However, these agents can cause side/adverse effects such as abdominal pain, flatulence, diarrhea and liver toxicity (Nazir et al., 2018). Berries have been studied for their ability to modulate sugars and fats digestion/breakdown through inhibition of key digestive enzymes, leading to delayed glycaemic response and reduced calorie intake (Edirisinghe \& Burton-Freeman, 2016; Ho, Nguyen, et al., 2017; McDougall et al., 2008, 2009; Worsztynowicz et al., 2014).
In this work, leaves were more potent than berries towards inhibition of $\alpha$-glucosidase (Table 3). No statistical differences were found between leaves extracts of both analysed species $(p<.05)$. Acarbose and 1-DNJ (1-deoxynojirimycin or moranoline), a natural $\alpha$-glucosidase inhibitor isolated from Morus spp. leaves (Borges de Melo, da Silveira Gomes, \& Carvalho, 2006), were the most active agents (Table 3).

Acarbose was the most active agent in the $\alpha$-amylase inhibitory assay (Table 3). A different potency was observed in this case: E. umbellata (leaves) $>$ berries (E. umbellata $>S$. lanceolata) $>S$. lanceolata (leaves).

In the case of lipase, the anti-obesity drug orlistat exhibited the most potent inhibitory activity (Table 3 ). In this assay, leaves (E. umbellata $>S$. lanceolata) were also more active than berries ( $S$. lanceolata $>$ E. umbellata).

In the present work, the inhibitory activity of carbohydrate- 


\section{Berries}

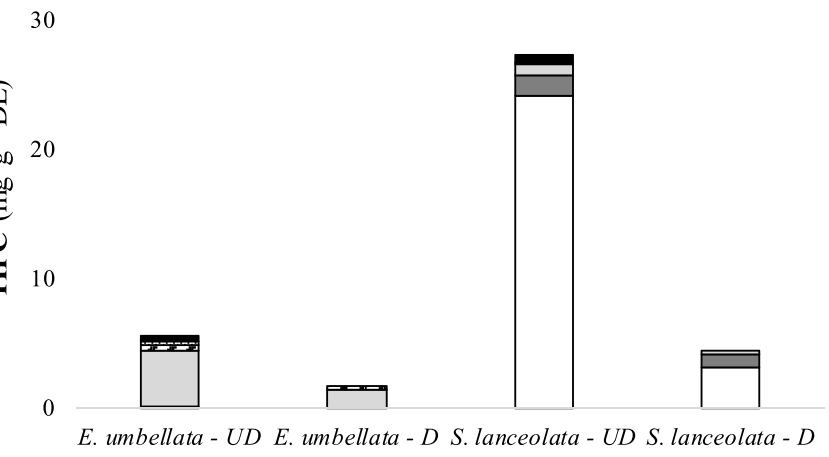

\section{Leaves}

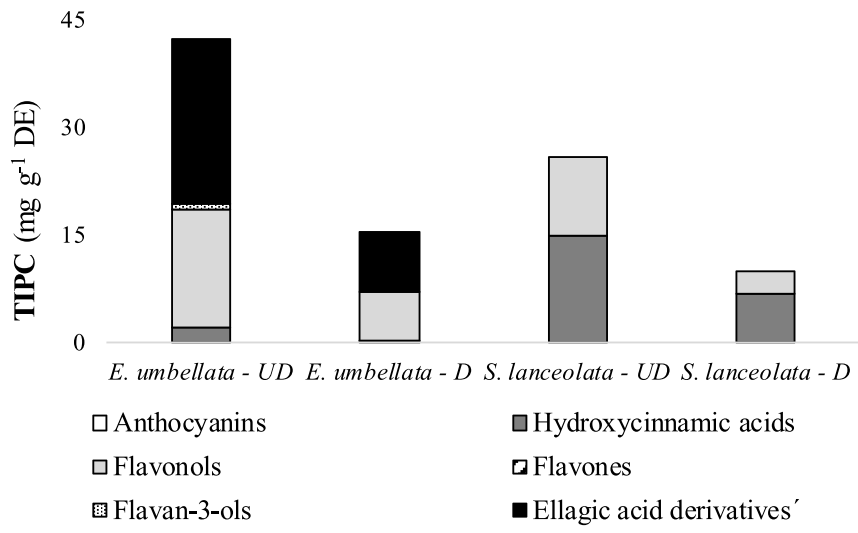

Fig. 1. Content of phenolic compounds $\left(\mathrm{mg} \mathrm{g}^{-1} \mathrm{DE}\right)$ of studied species before (Undigested) and after complete in vitro gastrointestinal digestion (Digested). TIPC: total individual phenolic content. UD: Undigested. D: Digested.

\section{Table 3}

In vitro inhibitory activities of studied species extracts towards digestive enzymes linked to sugars and fats metabolism. Results are expressed as the $\mathrm{IC}_{50}$ value $\left(\mathrm{mg} \mathrm{mL}^{-1}\right)$. Data represent the mean \pm standard deviation $(n=3)$.

\begin{tabular}{llll}
\hline & $\alpha$-Glucosidase & $\alpha$-Amylase & Lipase \\
\hline Berries & & & \\
E. umbellata & $6.02 \pm 0.23^{\mathrm{d}}$ & $4.01 \pm 0.20^{\mathrm{c}}$ & $9.68 \pm 0.51^{\mathrm{e}}$ \\
S. lanceolata & $7.55 \pm 0.27^{\mathrm{e}}$ & $6.01 \pm 0.54^{\mathrm{d}}$ & $7.75 \pm 0.48^{\mathrm{d}}$ \\
Leaves & & \\
$\begin{array}{l}\text { E. umbellata } \\
\text { S. lanceolata }\end{array}$ & $4.76 \pm 0.09^{\mathrm{c}}$ & $2.18 \pm 0.13^{\mathrm{b}}$ & $5.03 \pm 0.22^{\mathrm{b}}$ \\
$\begin{array}{l}\text { Reference compounds } \\
\text { Acarbose }\end{array}$ & $4.97 \pm 0.25^{\mathrm{c}}$ & $7.71 \pm 0.32^{\mathrm{e}}$ & $6.64 \pm 0.25^{\mathrm{c}}$ \\
1-DNJ & $0.12 \pm 0.01^{\mathrm{b}}$ & $0.02 \pm 0.01^{\mathrm{a}}$ & - \\
Orlistat & $0.01 \pm 0.01^{\mathrm{a}}$ & - & - \\
& & - & $0.47 \pm 0.02^{\mathrm{a}}$ \\
\end{tabular}

1-DNJ: 1-deoxynojirimycin; Means in the same column not sharing the same letter are significantly different at $p<.05$ probability level.

hydrolysing enzymes was not correlated with the TIPC of the analysed samples. Poor correlations $(r \leq-0.332)$ were also reported on a previous work (Podsędek et al., 2014), suggesting that the type of phenolics is more pertinent for the inhibitory effects towards digestive enzymes than the total phenolic amounts. The main enzymes involved in the final digestive step of carbohydrates are $\alpha$-amylase and $\alpha$-glucosidase (Grussu et al., 2011; Ho, Nguyen, et al., 2017; Podsędek et al., 2014). $\alpha$-Glucosidase is susceptible to a large variety of phenolic compounds (anthocyanins, caffeoylquinic acids (CQAs), flavonols) (McDougall et al., 2008; McDougall \& Stewart, 2005). Hence, the very distinct phenolic compositions of the analysed berry species (Fig. 1) could have dictated this outcome. Differences in composition and contents of phenolic compounds are known to affect their inhibitory activity towards enzymes. The large diversity in the structures between different groups of phenolic compounds, as well as within the same group, determines their ability to bind to digestive enzymes (Ho, Kase, et al., 2017; Podsędek et al., 2014). Additionally, phenolic compounds present in the extracts could act synergistically and/or antagonistically towards enzymes and influence the inhibitory activities (Grussu et al., 2011; Podsędek et al., 2014; Worsztynowicz et al., 2014). Previously (Ho, Kase, et al., 2017; Ho, Nguyen, et al., 2017), S. nigra (elderberry) berry extracts showed potent $\alpha$-glucosidase activities. Anthocyanins (cyanidin-3-O-sambubioside, cyanidin-3-O-glucoside, and cyanidin) and proanthocyanidins (B2, B5 and $\mathrm{C} 1$ ), isolated from berries, were reported as the main inhibitory agents (Ho, Kase, et al., 2017). Anthocyanins are important $\alpha$-glucosidase inhibitors (McDougall et al., 2008; McDougall \& Stewart, 2005). However, this study shows the lack of anthocyanins in E. umbellata (Table 3) and still a remarkable bioactivity. $M$. faya berries (with comparable anthocyanins content to $S$. lanceolata) displayed higher potencies (Spínola, Llorent-Martínez, \& Castilho, 2019). Berries were composed by cyanidin-3-O-glucoside, while $S$. lanceolata by cyanidin- $O$-sambubioside (a diglycoside). It seems that the nature of sugar moieties attached to the cyanidin aglycone modulate the bioactivity of anthocyanins. For example, cyanidin3,5-diglycoside had no inhibitory activity against $\alpha$-glucosidase, while cyanidin and its mono-glycosides were effective inhibitors (Akkarachiyasit, Charoenlertkul, Yibchok-Anun, \& Adisakwattana, 2010). Ellagitannins have a low impact on the activity of $\alpha$-glucosidase activity, while CQAs are strong inhibitors (McDougall \& Stewart, 2005; Meng, Cao, Feng, Peng, \& Hu, 2013). 3-O-CQA was dominant in $S$. lanceolata leaves (41\% of TIPC) (Pinto et al., 2017) and could justify the identical efficacy to $E$. umbellata leaves (Table 3 ), which are composed mainly by ellagitannins (56.83\%) (Table 2).

$\alpha$-Amylase activity is primarily affected by condensed (ellagitannins) and hydrolysable tannins (proanthocyanidins) (McDougall \& Stewart, 2005). In fact, E. umbellata (rich in ellagitannins) displayed the highest inhibitory activity (Table 3). Recently (Nazir et al., 2018), rutin, quercetin, and epigallocatechin gallate were the main contributors of $E$. umbellata berries against $\alpha$-amylase. Anthocyanins from $S$. nigra showed strong inhibition of $\alpha$-amylase (Ho, Kase, et al., 2017). This could partially justify the higher effect of $S$. lanceolata berries by comparison with respective leaves (Table 3). However, some authors (Grussu et al., 2011; Worsztynowicz et al., 2014) state that anthocyanins are not crucial for $\alpha$-amylase inhibition but their presence can potentiate the effectiveness of other phenolic compounds present in berries. Although no correlation was observed in the present study, these data could be indicative of the impact of these phenolic classes against $\alpha$-amylase activity.

Pancreatic lipase is responsible for the hydrolysis of $50-70 \%$ of dietary fats into monoacylglycerols and free fatty acids the intestinal lumen (McDougall et al., 2009). Inhibition of this enzyme is efficient in weight management and obesity control in diabetic patients (Podsędek et al., 2014). In the lipase assay, a strong correlation with TIPC was obtained $(r=-0.962)$, flavonols $(r=-0.852)$ and ellagitannins $(r=-0.806)$ being key inhibitors of lipase. The catabolic activity of lipase is more specific and ellagitannins are also reported to be the main inhibitors (McDougall et al., 2008, 2009). Anthocyanins (Worsztynowicz et al., 2014; You et al., 2011) and CQAs (Meng et al., 2013) are also reported inhibitors, which could justify the higher potencies of $S$. lanceolata samples compared to $E$. umbellata berries.

\subsection{In vitro inhibition of human aldose reductase (HAR)}

Most studies using phenolic compounds focus only at maintaining the glycaemic control, through inhibition of digestive enzymes. So, many of these hypoglycaemic agents have not been investigated for their beneficial effects on secondary complications of T2DM 
Table 4

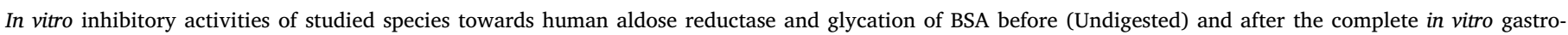
intestinal digestion (Digested). Results are expressed as the $\mathrm{IC}_{50}$ value $\left(\mathrm{mg} \mathrm{mL}^{-1}\right)$. Data represent the mean \pm standard deviation $(n=3)$.

\begin{tabular}{|c|c|c|c|c|c|c|}
\hline & \multicolumn{2}{|c|}{ Aldose reductase } & \multicolumn{2}{|c|}{ BSA glycation - ribose } & \multicolumn{2}{|c|}{ BSA glycation - fructose } \\
\hline & Undigested & Digested & Undigested & Digested & Undigested & Digested \\
\hline \multicolumn{7}{|l|}{ Berries } \\
\hline E. umbellata & $2.19 \pm 0.10^{\mathrm{c}}$ & $9.34 \pm 0.50^{g}$ & $5.35 \pm 0.21^{\mathrm{d}}$ & $9.34 \pm 0.21^{g}$ & $2.75 \pm 0.15^{\mathrm{d}}$ & $6.31 \pm 0.25^{g}$ \\
\hline S. lanceolata & $4.46 \pm 0.17^{\mathrm{f}}$ & $12.41 \pm 0.5 \mathrm{~h}$ & $6.04 \pm 0.30^{\mathrm{e}}$ & $11.21 \pm 0.25^{\mathrm{h}}$ & $4.10 \pm 0.15^{f}$ & $8.15 \pm 0.31^{\mathrm{h}}$ \\
\hline \multicolumn{7}{|l|}{ Leaves } \\
\hline E. umbellata & $0.86 \pm 0.02^{\mathrm{b}}$ & $2.51 \pm 0.10^{\mathrm{d}}$ & $2.38 \pm 0.10^{\mathrm{b}}$ & $5.69 \pm 0.22^{\mathrm{d}}$ & $1.22 \pm 0.05^{\mathrm{b}}$ & $3.25 \pm 0.14^{\mathrm{e}}$ \\
\hline S. lanceolata & $3.61 \pm 0.14^{\mathrm{e}}$ & $9.07 \pm 0.38^{g}$ & $4.47 \pm 0.16^{\mathrm{c}}$ & $7.41 \pm 0.33^{f}$ & $2.30 \pm 0.08^{\mathrm{c}}$ & $4.48 \pm 0.10^{f}$ \\
\hline \multicolumn{7}{|c|}{ Reference compounds } \\
\hline AMG & - & - & $9.56 \pm 0.36^{g}$ & - & $2.29 \pm 0.13^{\mathrm{c}}$ & - \\
\hline Quercetin & $0.10 \pm 0.01^{\mathrm{a}}$ & - & $0.11 \pm 0.01^{\mathrm{a}}$ & - & $0.24 \pm 0.02^{\mathrm{a}}$ & - \\
\hline
\end{tabular}

AMG: Aminoguanidine; N.I.: no inhibition. Means in the same assay not sharing the same letter are significantly different at $p<.05$ probability level.

(retinopathy, neuropathy, nephropathy, etc.), triggered by chronic hyperglycaemia. The overactivity of the polyol pathway is associated with the pathogenesis of diabetic complications (Grewal et al., 2016; Khangholi, Majid, Berwary, Ahmad, \& Aziz, 2016). In hyperglycaemia conditions, aldose reductase catalyses the reduction of excessive glucose to sorbitol, which is the first reaction of the polyol pathway. Then, sorbitol dehydrogenase reduces sorbitol to fructose, which intensifies to the formation of advanced glycation end-products (AGEs) (Grewal et al., 2016; Suryanarayana, Kumar, Saraswat, Petrash, \& Reddy, 2004). Hence, inhibition of the aldose reductase activity is another therapeutic target for the mitigation of T2DM signs (Grewal et al., 2016; Khangholi et al., 2016; Suryanarayana et al., 2004).

The analysed extracts interfered with the catabolic activity of HAR, although in lower extent than the quercetin standard (positive control) $(p<.05)$ (Table 4). E. umbellata extracts were the most active samples (leaves $>$ berries). A poor correlation $(r=-0.241)$ was found between TIPC and the reported bioactivities. Ellagitannins were the main active agents $(r=-0.838)$ for this assay and are documented as strong aldose reductase inhibitors (Suryanarayana et al., 2004).

After simulated gastrointestinal digestion, extracts presented a much lower inhibitory activity towards HAR $(p<.05)$ (Table 4). An increase of the $\mathrm{IC}_{50}$ values $(60-77 \%)$ was observed. This should be linked with the reduced amounts of phenolic compounds present in the digested extracts (Table 2). Correlations of HAR activity with TIPC improved after the digestion process $(r=-0.838)$, being flavonols $(r=-0.968)$ and ellagitannins $(r=-0.939)$ the compounds that contributed the most. Although in lower potency, phenolic compounds present in digested extracts still displayed inhibitory effects against HAR, meaning that they could potentially exert their beneficial effects on target tissues after digestion.

\subsection{In vitro inhibition of advanced glycation end-products (AGEs) generation}

The elevated blood glucose levels can also cause glycation of proteins (albumin collagen, elastin), and consequently, accumulation of AGEs in human tissues (Bains \& Gugliucci, 2017; Khangholi et al., 2016; Yeh et al., 2017). Also, the excessive production of fructose, though the overactivated polyol pathway, intensifies the generation of AGEs (Wang, Yagiz, Buran, Nunes, \& Gu, 2011). These adducts are originated via binding of carbonyl groups of reducing sugars (glucose, fructose, ribose) to free amino groups of proteins (Maillard reaction). The first glycation product is the highly reversible Schiff base intermediates (glucosamines), which are further converted to more stable Amadori products. These molecules undergo a series of reactions resulting in carbonyl compounds (glyoxal, methylglyoxal, 3-deoxyglucosone) (Beaulieu et al., 2010; Khangholi et al., 2016). AGEs lead to structural and functional changes in cellular and tissues components (proteins, lipids, DNA), being implicated in the pathogenesis of angiopathy, neuropathy, nephropathy, etc. in diabetic patients (Yeh et al., 2017). Therefore, inhibition of protein glycation may be an alternative therapeutic approach for delaying and averting some diabetic complications (Beaulieu et al., 2010; Harris et al., 2014; Wang et al., 2011).

The analysed extracts were found to prevent protein glycation, although with different potencies between sugar models (Table 4). In the case of ribose as glycation agent, samples were more active than aminoguanidine (AMG), a synthetic AGEs inhibitor. Leaves displayed higher inhibitory activities than berries; E. umbellata showing the strongest results (Table 4). When fructose was used, only E. umbellata leaves were more active than AMG $(p<.05)$. Leaves of $S$. lanceolata showed a comparable result to that of AMG, while berries were less potent (E. umbellata $>$ S. lanceolata) (Table 4). Quercetin standard displayed the best inhibitory results (in both models) (Table 4). A similar trend was documented before using pure quercetin as positive control (Beaulieu et al., 2010; Harris et al., 2014). Samples were more active in the fructose model (lower $\mathrm{IC}_{50}$ values) than in ribose. The reactivity of individual sugars depends largely on the proportion that exists in the open-chain form (Harding \& Ganea, 2006). D-ribose is the most reactive reducing sugar in the glycation of proteins due to its unstable aldofuranose ring (two $\mathrm{OH}$ in axial position) (Bains \& Gugliucci, 2017; Wei et al., 2012). Hence, the abundance of ribose in open chain form is higher than fructose, the former being more vulnerable to reactions with amino groups of proteins (Wei et al., 2012).

Inhibition of BSA glycation was poorly correlated with TIPC $(r \leq-0.546)$. From a parallel works (Spínola et al., 2018; Spínola, Llorent-Martínez, \& Castilho, 2019; Spínola, Pinto, et al., 2019), species with higher TIPC (M. faya, R. grandifolius and Vaccinium spp.) were more effective towards inhibition of AGEs formation than analysed extracts. In the present study, flavonols played a determinant role in the prevention of AGEs generation ( $r \geq-0.958$ ); they are reported to be effective anti-glycative compounds (Yeh et al., 2017).

Upon digestion, the analysed extracts were still able to prevent the in vitro glycation of BSA (Table 5), albeit in lower potency $(p<.05)$. The increase of IC $_{50}$ values (40-62\%) suggested that digested extracts showed lower inhibitory activity against formation of AGEs. Again, the reduced levels of phenolic compounds in the digested extracts (Table 2) is associated with this outcome. After digestion, correlation was improved with TIPC ( $r \geq-0.845$ ); flavonols remained the main contributors for these bioactivities $(r \geq-0.944)$. Bains and Gugliucci (2017) showed that formation of fructose-AGEs in the enteral lumen occurs after consumption of fructose-rich foodstuffs. Hence, the antiglycative effects of phenolic compounds can prevent the generation of AGEs, which, after being absorbed may contribute to inflammatory diseases. This data together with inhibition of HAR (section 3.4), indicates another potential therapeutic mechanism of analysed species against long-term diabetic complications, besides lowering 
Table 5

In vitro antioxidant activities of $E$. umbellata methanolic extracts (berries and leaves) before (Undigested) and after the complete in vitro gastrointestinal digestion (Digested). Results are expressed as mmol Trolox equivalents per $g$ of dry extract $\left(\mathrm{mmol} \mathrm{TE} \mathrm{g}^{-1} \mathrm{DE}\right)$. Data represent the mean \pm standard deviation $(n=3)$.

\begin{tabular}{|c|c|c|c|c|}
\hline & \multicolumn{2}{|l|}{ Berries } & \multicolumn{2}{|l|}{ Leaves } \\
\hline & Undigested & Digested & Undigested & Digested \\
\hline $\mathrm{ABTS}^{+\cdot}$ & $0.68 \pm 0.02^{b}$ & $0.17 \pm 0.01^{\mathrm{a}}$ & $4.71 \pm 0.18^{\mathrm{d}}$ & $1.90 \pm 0.04^{\mathrm{c}}$ \\
\hline DPPH ${ }^{*}$ & $0.13 \pm 0.01^{\mathrm{a}}$ & $0.14 \pm 0.01^{\mathrm{b}}$ & $0.84 \pm 0.02^{\mathrm{d}}$ & $0.33 \pm 0.01^{\mathrm{c}}$ \\
\hline $\mathrm{NO}^{\circ}$ & $0.08 \pm 0.01^{\mathrm{b}}$ & $0.02 \pm 0.01^{\mathrm{a}}$ & $0.39 \pm 0.01^{\mathrm{d}}$ & $0.14 \pm 0.01^{\mathrm{c}}$ \\
\hline $\mathrm{O}_{2}^{*-}$ & $0.03 \pm 0.01^{\mathrm{b}}$ & $0.01 \pm 0.01^{\mathrm{a}}$ & $0.22 \pm 0.01^{\mathrm{d}}$ & $0.09 \pm 0.01^{\mathrm{c}}$ \\
\hline
\end{tabular}

Means in the same line not sharing the same letter are significantly different at $p<.05$ probability level.

hyperglycaemia through modulation of carbohydrate-hydrolysing enzymes activity (section 3.3).

\subsection{In vitro antioxidant assays}

Hyperglycaemia intensifies the production of reactive oxygen species (ROS) that have been implicated as a contributing factor in the development and progression of T2DM (Grewal et al., 2016; Ho, Kase, et al., 2017; Ho, Nguyen, et al., 2017). Berries are reported to possess high antioxidant activities due to its considerable amounts of phenolic compounds (Bermúdez-Soto et al., 2007; Edirisinghe \& BurtonFreeman, 2016). Antioxidants, such as phenolic compounds, poses hypoglycaemic effects through at least two means, by inhibiting oxidative formation of AGEs and by scavenging ROS (Beaulieu et al., 2010; Yeh et al., 2017). Hence, dietary intake of berries seems beneficial to prevent oxidative damage and diabetic complications (Edirisinghe \& Burton-Freeman, 2016; Podsędek et al., 2014).

The antioxidant activity of $E$. umbellata extracts was assessed by four in vitro assays, using synthetic $\left(\mathrm{ABTS}^{+}{ }^{+}, \mathrm{DPPH}^{*}\right)$ and biological radicals $\left(\mathrm{NO}^{\circ}, \mathrm{O}_{2}^{-\bullet}\right.$ ).

Leaves showed the best results among all assays (Table 5). The scavenging activities of $E$. umbellata extracts (berries and leaves) have been reported (Ishaq et al., 2015; Khattak, 2012; Kim et al., 2016; Nazir et al., 2018; Ozen et al., 2017). However, the results are expressed as the $\mathrm{IC}_{50}$ values, which make it difficult to establish a comparison with the present data. Nevertheless, leaves showed stronger antioxidant activities than berries (Kim et al., 2016), which agrees with the present results. By comparison, $S$. lanceolata berries (analysed under the same conditions) (Pinto et al., 2017) displayed higher overall activities than E. umbellata counterparts. It seems that the present antioxidant activities are related to the total phenolic contents of analysed extracts, since samples with the highest TIPC showed, in general, the greatest antioxidant activities $(r \geq 0.805)$. Flavonols $(r \geq 0.913)$ and ellagitannins $(r \geq 0.826)$ were the main contributors for the obtained results. In fact, R. grandifolius, M. faya and Vaccinium spp. with higher flavonols and/or ellagitannins contents, displayed superior antioxidant activities than analysed species (Spínola et al., 2018; Spínola, Llorent-Martínez, \& Castilho, 2019; Spínola, Pinto, et al., 2019).

Most studies that evaluate the antioxidant activity of berries do not consider the intensive metabolism and chemical alterations occurring during digestion, with a consequential impact on their bioactivity. This work demonstrates that the physiochemical changes occurring in the gastrointestinal tract should be considered when evaluating the potential antioxidant activity (Tagliazucchi et al., 2010). The lower antioxidant activities noted after digestion (Table 5) are probably the result of the lower phenolic content in the digested samples (Table 3). Similar results were also reported in other studies (Correa-Betanzo et al., 2014; Huang et al., 2014; Olejnik et al., 2016; Zhang et al., 2016), concluding that the digestion contributed to the loss of antioxidant capacity of berry-producing plants. Correlations between the antioxidant assays and TIPC were enhanced after the in vitro digestion $(r \geq-0.875)$. Ellagitannins $(r \geq 0.920)$ and flavonols $(r \geq 0.902)$ remained the most active compounds.

Since phenolic compounds are highly sensitive to the mild-alkaline conditions in the intestinal step, during gastrointestinal digestion, they undergo structural modifications and/or degradation and their bioactivities are affected (Bermúdez-Soto et al., 2007; Correa-Betanzo et al., 2014). The high pH values in the intestinal phase may induce deprotonation of the hydroxyl groups of phenolic compounds, making them unavailable to react with free radicals (Tagliazucchi et al., 2010). In addition, phenolic compounds may also interact with other constituents of the sample (proteins, lipids, fibers, polysaccharides), making them unavailable to react with free radicals (Bermúdez-Soto et al., 2007; Correa-Betanzo et al., 2014). Nevertheless, despite extensive degradation following digestion, analysed extracts still showed the capacity to scavenge free radicals (although in a minor extent). Whether or not the phenolic compounds are absorbed in the gut, the ones that maintain their antioxidant activity, after digestion, might carried out their potential beneficial effects in the gastrointestinal tract by scavenging ROS (Grussu et al., 2011; Liang et al., 2012; Tagliazucchi et al., 2010).

\section{Conclusions}

In this study, the enzyme-inhibitory properties and the antioxidant activities of two berry-producing plants $-E$. umbellata and $S$. lanceolata - have been studied and discussed in terms of their phenolic composition. It was observed that the analysed extracts presented effective inhibitory activities against key enzymes linked to T2DM and obesity. The analysed species were also able to prevent protein glycation and scavenge free radicals, highlighting their use as natural sources of biologically active compounds (in particular ellagitannins and flavonols). The gastrointestinal digestion simulation affected the phenolic content of $E$. umbellata and $S$. lanceolata. These changes were correlated with the decrease of in vitro anti-diabetic and antioxidant activities. However, despite these modifications, this study highlights the use of both species as dietary hypoglycaemic, anti-glycation and antioxidant agents (more pronounced for E. umbellata). It also demonstrates that bioactive compounds in leaves are more abundant that in berries and that anthocyanins, albeit important, are not the most relevant components when other substances, such as flavonoids and/or ellagic acid derivatives are available.

\section{Notes}

The authors declare no conflicts of interest.

\section{Acknowledgments}

The authors are grateful to Esmeralda Dias from "A Quintinha das Aromáticas" (Funchal, Portugal) for the supply of E. umbellata samples and to Francisco Fernandes and José Carvalho from Madeira Botanical Garden (Funchal) for the identification of plant material. V. Spínola acknowledges Fundação para a Ciência e a Tecnologia (FCT, Portugal) for a Ph.D. grant SFRH/BD/84672/2012. This work was also supported by FCT (project PEst-OE/QUI/UI0674/2019, CQM, Portuguese Government funds) and the Portuguese National Mass Spectrometry Network (Contract RNEMREDE/1508/REM/2005), and through Madeira 14-20 Program, project PROEQUIPRAM - Reforço do Investimento em Equipamentos e Infraestruturas Científicas na RAM (M1420-01-0145-FEDER-000008) and by ARDITI-Agência Regional para o Desenvolvimento da Investigação Tecnologia e Inovação, through the project M1420-01-0145-FEDER-000005 - Centro de Química da Madeira - $\mathrm{CQM}^{+}$(Madeira 14-20). 


\section{Appendix A. Supplementary data}

Supplementary data to this article can be found online at https:// doi.org/10.1016/j.foodres.2019.04.030.

\section{References}

Akkarachiyasit, S., Charoenlertkul, P., Yibchok-Anun, S., \& Adisakwattana, S. (2010). Inhibitory activities of cyanidin and its glycosides and synergistic effect with acarbose against intestinal $\alpha$-glucosidase and pancreatic $\alpha$-amylase. International Journal of Molecular Sciences, 11(9), 3387-3396.

Bains, Y., \& Gugliucci, A. (2017). Ilex paraguariensis and its main component chlorogenic acid inhibit fructose formation of advanced glycation endproducts with amino acids at conditions compatible with those in the digestive system. Fitoterapia, 117, 6-10.

Beaulieu, L.-P., Harris, C. S., Saleem, A., Cuerrier, A., Haddad, P. S., Martineau, L. C., ... Arnason, J. T. (2010). Inhibitory effect of the Cree traditional medicine Wiishichimanaanh (Vaccinium vitis-idaea) on advanced glycation endproduct formation: Identification of active principles. Phytotherapy Research, 24(5), 741-747.

Bermúdez-Soto, M.-J., Tomás-Barberán, F.-A., \& García-Conesa, M.-T. (2007). Stability of polyphenols in chokeberry (Aronia melanocarpa) subjected to in vitro gastric and pancreatic digestion. Food Chemistry, 102(3), 865-874.

Borges de Melo, E., da Silveira Gomes, A., \& Carvalho, I. (2006). $\alpha$ - and $\beta$-Glucosidase inhibitors: Chemical structure and biological activity. Tetrahedron, 62(44), 10277-10302.

Correa-Betanzo, J., Allen-Vercoe, E., McDonald, J., Schroeter, K., Corredig, M., \& Paliyath, G. (2014). Stability and biological activity of wild blueberry (Vaccinium angustifolium) polyphenols during simulated in vitro gastrointestinal digestion. Food Chemistry, 165, 522-531.

Edirisinghe, I., \& Burton-Freeman, B. (2016). Anti-diabetic actions of berry polyphenols Review on proposed mechanisms of action. Journal of Berry Research, 6(2), 237-250.

Ferlemi, A.-V., \& Lamari, F. (2016). Berry leaves: An alternative source of bioactive natural products of nutritional and medicinal value. Antioxidants, 5(17) (20 pp.).

Grewal, A. S., Bhardwaj, S., Pandita, D., Lather, V., \& Sekhon, B. S. (2016). Updates on aldose reductase inhibitors for management of diabetic complications and non-diabetic diseases. Mini-Reviews in Medicinal Chemistry, 16(2), 120-162.

Grussu, D., Stewart, D., \& McDougall, G. J. (2011). Berry polyphenols inhibit $\alpha$-amylase in vitro: Identifying active components in rowanberry and raspberry. Journal of Agricultural and Food Chemistry, 59(6), 2324-2331.

Guerra, A., Etienne-Mesmin, L., Livrelli, V., Denis, S., Blanquet-Diot, S., \& Alric, M. (2012). Relevance and challenges in modeling human gastric and small intestinal digestion. Trends in Biotechnology, 30(11), 591-600.

Harding, J. J., \& Ganea, E. (2006). Protection against glycation and similar post-translational modifications of proteins. Biochimica et Biophysica Acta - Proteins and Proteomics, 1764, 1436-1446.

Harris, C. S., Cuerrier, A., Lamont, E., Haddad, P. S., Arnason, J. T., Bennett, S. A. L., \& Johns, T. (2014). Investigating wild berries as a dietary approach to reducing the formation of advanced glycation endproducts: Chemical correlates of in vitro antiglycation activity. Plant Foods for Human Nutrition, 69(1), 71-77.

Ho, G. T. T., Kase, E. T., Wangensteen, H., \& Barsett, H. (2017). Phenolic elderberry extracts, anthocyanins, procyanidins, and metabolites influence glucose and fatty acid uptake in human skeletal muscle cells. Journal of Agricultural and Food Chemistry, 65(13), 2677-2685.

Ho, G. T. T., Nguyen, T. K. Y., Kase, E. T., Tadesse, M., Barsett, H., \& Wangensteen, H. (2017). Enhanced glucose uptake in human liver cells and inhibition of carbohydrate hydrolyzing enzymes by Nordic berry extracts. Molecules, 22(11), 1806-1821.

Huang, H., Sun, Y., Lou, S., Li, H., \& Ye, X. (2014). In vitro digestion combined with cellular assay to determine the antioxidant activity in Chinese bayberry (Myrica rubra Sieb. et Zucc.) fruits: A comparison with traditional methods. Food Chemistry, 146, 363-370.

Ishaq, S., Rathore, H. A., Sabir, S. M., \& Maroof, M. S. (2015). Antioxidant properties of Elaeagnus umbellata berry solvent extracts against lipid peroxidation in mice brain and liver tissues. Food Science and Biotechnology, 24(2), 673-679.

Khangholi, S., Majid, F. A. A., Berwary, N. J. A., Ahmad, F., \& Aziz, R. B. A. (2016). The mechanisms of inhibition of advanced glycation end products formation through polyphenols in hyperglycemic condition. Planta Medica, 82, 32-45.

Khattak, K. F. (2012). Free radical scavenging activity, phytochemical composition and nutrient analysis of Elaeagnus umbellata berry. Journal of Medicinal Plants Research, 6(39), 5196-5203.

Kim, M., Lim, J., \& Yang, S. (2016). Component analysis and anti-proliferative effects of ethanol extracts of fruits, leaves, and stems from Elaeagnus umbellata in HepG2 cells.
Journal of Korean Society of Food Science and Nutrition, 45(6), 828-834.

Liang, L., Wu, X., Zhao, T., Zhao, J., Li, F., Zou, Y., ... Yang, L. (2012). In vitro bioaccessibility and antioxidant activity of anthocyanins from mulberry (Morus atropurpurea Roxb.) following simulated gastro-intestinal digestion. Food Research International, 46(1), 76-82.

McDougall, G. J., Kulkarni, N. N., \& Stewart, D. (2008). Current developments on the inhibitory effects of berry polyphenols on digestive enzymes. BioFactors, 34(1), 73-80.

McDougall, G. J., Kulkarni, N. N., \& Stewart, D. (2009). Berry polyphenols inhibit pancreatic lipase activity in vitro. Food Chemistry, 115, 193-199.

McDougall, G. J., \& Stewart, D. (2005). The inhibitory effects of berry polyphenols on digestive enzymes. BioFactors, 23(4), 189-195.

Meng, S., Cao, J., Feng, Q., Peng, J., \& Hu, Y. (2013). Roles of chlorogenic acid on regulating glucose and lipids metabolism: A review. Evidence-based Complementary and Alternative Medicine Article ID 801457, 11 pages.

Nazir, N., Zahoor, M., Nisar, M., Khan, I., Karim, N., Abdel-Halim, H., \& Ali, A. (2018) Phytochemical analysis and antidiabetic potential of Elaeagnus umbellata (Thunb.) in streptozotocin-induced diabetic rats: Pharmacological and computational approach. BMC Complementary and Alternative Medicine, 18(1), 1-16. https://doi.org/10.1186/ s12906-018-2381-8.

Olejnik, A., Olkowicz, M., Kowalska, K., Rychlik, J., Dembczyński, R., Myszka, K., .. Moyer, M. P. (2016). Gastrointestinal digested Sambucus nigra L. fruit extract protects in vitro cultured human colon cells against oxidative stress. Food Chemistry, 197, 648-657.

Ozen, T., Yenigun, S., Altun, M., \& Demirtas, I. (2017). Phytochemical constituents, ChEs and urease inhibitions, antiproliferative and antioxidant properties of Elaeagnus umbellata Thunb. Combinatorial Chemistry \& High Throughput Screening, 20(6), 558-577.

Pei, R., Yu, M., Bruno, R., \& Bolling, B. W. (2015). Phenolic and tocopherol content of autumn olive (Elaeagnus umbellate) berries. Journal of Functional Foods, 16, 305-314.

Pinto, J., Spínola, V., Llorent-Martínez, E. J., Fernández-de Córdova, M. L., MolinaGarcía, L., \& Castilho, P. C. (2017). Polyphenolic profile and antioxidant activities of Madeiran elderberry (Sambucus lanceolata) as affected by simulated in vitro digestion. Food Research International, 100(P3), 404-410.

Podsędek, A., Majewska, I., Redzynia, M., Sosnowska, D., \& Koziołkiewicz, M. (2014). In vitro inhibitory effect on digestive enzymes and antioxidant potential of commonly consumed fruits. Journal of Agricultural and Food Chemistry, 62(20), 4610-4617.

Spínola, V., Llorent-Martínez, E. J., \& Castilho, P. C. (2019). Polyphenols of Myrica faya inhibit key enzymes linked to type II diabetes and obesity and formation of advanced glycation end-products (in vitro): Potential role in the prevention of diabetic complications. Food Research International, 116, 1229-1238.

Spínola, V., Pinto, J., \& Castilho, P. C. (2018). Hypoglycemic, anti-glycation and antioxidant in vitro properties of two Vaccinium species from Macaronesia: A relation to their phenolic composition. Journal of Functional Foods, 40, 595-605.

Spínola, V., Pinto, J., Llorent-Martínez, E. J., Tomás, H., \& Castilho, P. C. (2019). Evaluation of Rubus grandifolius L. (wild blackberries) activities targeting management of type-2 diabetes and obesity using in vitro models. Food and Chemical Toxicology, 123, 443-452 September 2018.

Suryanarayana, P., Kumar, P. A., Saraswat, M., Petrash, J. M., \& Reddy, G. B. (2004). Inhibition of aldose reductase by tannoid principles of Emblica officinalis: Implications for the prevention of sugar cataract. Molecular Vision, 10, 148-154.

Tagliazucchi, D., Verzelloni, E., Bertolini, D., \& Conte, A. (2010). In vitro bio-accessibility and antioxidant activity of grape polyphenols. Food Chemistry, 120(2), 599-606.

Wang, W., Yagiz, Y., Buran, T. J., Nunes, C. D. N., \& Gu, L. (2011). Phytochemicals from berries and grapes inhibited the formation of advanced glycation end-products by scavenging reactive carbonyls. Food Research International, 44(9), 2666-2673.

Wei, Y., Han, C. S., Zhou, J., Liu, Y., Chen, L., \& He, R. Q. (2012). D-ribose in glycation and protein aggregation. Biochimica et Biophysica Acta, 1820, 488-494.

Worsztynowicz, P., Napierała, M., Białas, W., Grajek, W., \& Olkowicz, M. (2014). Pancreatic $\alpha$-amylase and lipase inhibitory activity of polyphenolic compounds present in the extract of black chokeberry (Aronia melanocarpa L.). Process Biochemistry, 49(9), 1457-1463.

Yeh, W., Hsia, S., Lee, W., \& Wu, C. (2017). Polyphenols with antiglycation activity and mechanisms of action: A review of recent findings. Journal of Food and Drug Analysis, 25, 84-92.

You, Q., Chen, F., Wang, X., Luo, P. G., \& Jiang, Y. (2011). Inhibitory effects of Muscadine anthocyanins on $\alpha$-glucosidase and pancreatic lipase activities. Journal of Agricultural and Food Chemistry, 59(17), 9506-9511.

Zhang, Y., Chen, S., Wei, C., Gong, H., Li, L., \& Ye, X. (2016). Chemical and cellular assays combined with in vitro digestion to determine the antioxidant activity of flavonoids from Chinese bayberry (Myrica rubra Sieb. et Zucc.) leaves. PLoS One, 11(12), e0167484. 